\title{
Drug Development for Asthma and COPD: A Regulatory Perspective
}

\author{
Marianne Mann MD and Robert J Meyer MD
}

\author{
Introduction \\ General Regulatory Overview \\ Asthma Drug Development Overview \\ Asthma Drug Development by Class \\ COPD Drug Development Overview \\ COPD Drug Development by Class \\ Generic Inhaled Drug Development for COPD and Asthma \\ Summary
}

\begin{abstract}
This review addresses drug development intended to support United States clearance for asthma and COPD by explaining basic regulatory terms and broadly discussing the regulatory pathways to clearance. Some of the key clinical regulatory challenges that are faced by drugs for asthma and COPD are explained and clarified, both overall and by class of drug, citing relevant examples that emphasize key lessons. Generic drug development of inhaled drugs is also addressed. The purpose of this review is to provide the reader with a greater understanding and appreciation of asthma and COPD drug development from the regulatory perspective. Key words: COPD; asthma; drug development; regulatory. [Respir Care 2018;63(6):797-817. () 2018 Daedalus Enterprises]
\end{abstract}

\section{Introduction}

The development of drugs for asthma and COPD in the United States involves regulatory oversight and clearance, the specifics of which are not familiar to many prescribers. While clinical guidelines and individual prescribers rec-

\footnotetext{
Dr Mann is an independent clinical/regulatory drug development consultant in Highland, Maryland. Dr Meyer is affiliated with Greenleaf Health, Washington, DC and the University of Virginia, Charlottesville, Virginia.

Drs Mann and Meyer both serve as regulatory consultants to industry to provide clinical and regulatory input to facilitate drug development. Confidentiality agreements preclude naming these companies. Dr Meyer discloses a relationship with Cardiome Pharma. Dr Mann discloses a relationship with Juniper Pharmaceuticals. Previously, both Drs Mann and Meyer worked at the Food and Drug Administration in the Division of Pulmonary and Allergy Drug Products, but they do not represent that agency in this article and all opinions in this paper are their own. Dr Meyer's opinions do not necessarily represent those of the University of Virginia or the Commonwealth of Virginia.
}

Dr Mann presented a version of this paper at the 56th RESPIRATORY CARE Journal Conference, Respiratory Medications for COPD and Adult Asthma: Pharmacologic Actions to Clinical Applications, held June 22-23, 2017 in St Petersburg, Florida. ommend the use of products for asthma or COPD on the basis of evolving clinical data and experience, the U.S. Food and Drug Administration (FDA) clearance of drugs is based on regulatory and legal standards, including the need for sponsors to provide substantial evidence for effectiveness. The FDA follows the rules and regulations set forth in the Code of Federal Regulations (CFR), which implements the Food, Drug, and Cosmetics Act. Substantial evidence for efficacy is determined in 21 CFR 314.126 based on reports of adequate and well-controlled investigations. Generally, this is interpreted as at least 2 pivotal trials that show clear evidence of efficacy based on clinically relevant end points of efficacy. Even with this standard met, the FDA must weigh all of the clinical, non-clinical, and quality data (ie, chemistry and manufacturing information)

Correspondence: Marianne Mann MD, 7105 Biter Lane Highland, MD 20777. E-mail: mann612@verizon.net or Robert J Meyer MD, 1055 Thomas Jefferson Street, NW, Suite 450 Washington, DC 20007. E-mail: rjm7cd@virginia.edu.

DOI: $10.4187 /$ respcare.06009 


\section{Drug Development for Asthma AND COPD}

for a product to determine its overall benefit/risk. The clearance of a drug and the resulting labeling reflects the key evidence provided to and reviewed by the FDA.

The purpose of this review is to describe the development of drugs for asthma and COPD intended for marketing in the United States, and to help clarify the types of well-controlled clinical investigations that are necessary for regulatory clearance. This review will also address briefly the development of generic inhaled drugs. This review will give practitioners a fuller understanding of drug development for COPD and asthma.

\section{General Regulatory Overview}

Development for any therapeutic drug intended for marketing in the United States begins with an Investigational New Drug application that is filed with the FDA to allow studies in humans to proceed. Drug development proceeds in several phases, although these do not need to be distinct or serial. Phase 1 studies are intended to explore the pharmacokinetics $(\mathrm{PK})$ and tolerability profile of the drug in a relatively small number of healthy volunteer subjects (or, less commonly, patients). Later PK studies may involve special populations (eg, renal impairment) or explore drugdrug interactions. Phase 2 studies enroll well-defined subjects with the intent to explore efficacy, establish the dose range/or regimen, and further delineate safety and tolerability. Phase 3 studies are large, randomized, controlled clinical trials that establish the safety and efficacy of the drug for the desired indication and are often referred to as pivotal studies. Phase 4 studies are post-clearance studies that are done to further clarify the safety profile and/or the efficacy of the drug, often in new subject groups or for related indications. Once the range of phase 1-3 studies are complete, applicants submit a New Drug Application (NDA) for a small molecule drug or a Biologic License Application for a product produced in a biologic system, such as a monoclonal antibody. These applications include not only the clinical data from the phase 1,2 , and 3 studies, but also include data on product quality, manufacturing, and animal toxicology/pharmacology. While timing may vary, it generally takes $10-13$ y for a new medicine to go from initial discovery to the marketplace, with clinical trials alone taking $6-7 \mathrm{y} .{ }^{1}$

The CFR outlines the many rules by which the FDA guides drug development, but the interpretation of those rules is what is most important to guiding drug development. Many helpful guidances related to drug development are available on the FDA Website. ${ }^{2}$ The federal Food, Drug, and Cosmetic Act Drug Efficacy Amendment of 1962 requires that the effectiveness of products be demonstrated through adequate and well-controlled clinical trials. In general, at least 2 adequate and well-controlled phase 3 trials are required for clearance. A 1998 FDA
Guidance entitled Providing Evidence of Effectiveness for Human Drug and Biological Products ${ }^{3}$ further clarifies what types of studies should be done to support an FDA clearance and addresses special situations in which a single pivotal trial may provide sufficient evidence for efficacy.

The FDA's regulations on adequate and well-controlled studies (21 CFR 314.126) describe the types of controlled trials that best provide evidence of effectiveness. Placebocontrolled trials are done most often because superiority trials are in many ways the most robust in design. Importantly, these trials are often performed upon a background standard of care, and subjects randomized to placebo receive placebo plus effective standard of care therapy, while subjects randomized to active therapy have this added to the standard of care, as long as it is not contraindicated with the study drug. Criticism of the ethics of placebocontrolled trials has been raised, but sometimes it is not understood or appreciated that with a thoughtful design, placebo does not equate to no therapy. A new long-acting muscarinic antagonist (LAMA), for instance, can be evaluated versus placebo in subjects with COPD who are taking long-acting $\beta$ agonists (LABA), inhaled corticosteroids (ICS), and rescue albuterol, as needed.

Another way to study a drug and show efficacy is to perform dose-response trials in which a higher dose of the drug is demonstrated to be superior to a lower dose. A superiority trial that shows improved efficacy of the new test drug over an existing therapy is also a rational and accepted additional study design that can be considered, and is sometimes considered when placebo-controlled trials are unethical. In certain situations, the FDA will consider a trial in which subjects are exposed to the new drug and results are compared to historical data. These trials are considered in conditions, for instance, in which there is a high mortality rate and the drug therapy proposes to dramatically change that known mortality rate, or for conditions in which the desired effect (anesthesia for instance) is well known and understood. Non-inferiority trials comparing a new drug to a cleared reference drug can be considered, yet these are generally done only when superiority designs are not feasible or ethical. Many question why the FDA cannot rely more on non-inferiority trials to substantiate efficacy, as such studies would be easier to enroll, and would not pose as many ethical concerns.

A 2016 FDA guidance entitled Non-Inferiority Clinical Trials to Establish Effectiveness ${ }^{4}$ describes non-inferiority studies and why they are generally less desirable than superiority trial designs. While evidence of superiority in a clinical trial is "entirely interpretable without further assumptions (other than lack of bias) ... the non-inferiority study is dependent on knowing something that is not measured in the study, namely, that the active control had its expected effect." Non-inferiority trials rely heavily on trusting that the active comparator worked and that it would 
have beaten placebo by a predictable margin, had a placebo arm been in the study. For many cleared asthma and COPD drugs, it is appreciated that their effect sizes versus placebo can vary from trial to trial, and may, at times, even fail to distinguish from placebo. As per 21 CFR 314.126(a)(2)(iv), non-inferiority trials are challenging to interpret because similarity of test drug and active control can mean either that both drugs were effective or that neither was effective. Due to these challenges, whenever it is possible, the FDA prefers superiority trial designs to clearly demonstrate efficacy.

The legal pathways for a new prescription drug clearance include 3 regulatory pathways: the 505(b)(1) NDA, the 505(b)(2) NDA, and the 505(j) abbreviated NDA pathway. The 505(b)(1) NDA is the pathway used for new chemical entities in which the entire development program, including all preclinical studies, carcinogenicity studies, and a full clinical program are done by the sponsor (or the sponsor buys the rights to the data). The 505(b)(2) NDA drug application is a very common pathway that applies to drugs that have already be cleared but are now being formulated or delivered in a new way, or being submitted for a new indication. For instance, a LAMA already cleared for COPD is now seeking clearance for asthma, or a LABA that is delivered via a pressurized metered-dose inhaler (pMDI) is now to be delivered via a dry powder inhaler (DPI). In such cases, the 505(b)2 clinical development program would apply. The requirements for clearance under a 505(b)2 pathway generally remain substantial for inhaled drugs, particularly because topically active drugs need to re-establish their dose and regimen via the new device delivery system. The majority of new versions of previously cleared inhaled drugs for asthma and COPD have been 505(b)(2) applications; however, recently published guidances from the Office of Generic Drugs at the FDA have prompted more sponsors to consider the 505(j) abbreviated NDA route. The 505(j) abbreviated NDA route is for generic drugs, which are pharmaceutically and therapeutically equivalent to a referenced, previously cleared drug that is no longer protected by patents or exclusivity. These generic products are considered substitutable to the already cleared reference drug. The FDA provides for expedited review options for 505(b)1 or 505(b) 2 drugs that meet critical public health needs, and guidances are available that outline these various options..$^{5,6}$

\section{Asthma Drug Development Overview}

As of July 2017, the FDA has no guidance documents available that specifically address the development of drugs or biologics to treat asthma. In part, this likely reflects the relatively robust development of asthma therapies in recent decades and a general knowledge by drug developers of the FDA's regulatory expectations, memorialized in the
FDA's published action summaries and its public advisory committees. As the underlying pathology of asthma became better elucidated in the latter part of the last century and drove therapeutic guidelines (eg, the National Asthma Education and Prevention Program), the FDA viewed the development of asthma drugs broadly by general modes of action: bronchodilators (short-acting "quick relievers" and long-acting maintenance therapies) and anti-inflammatories (eg, corticosteroids, leukotriene antagonists). Inhaled therapy is considered to be preferred route of delivery for drugs specifically targeting the airways, particularly for bronchodilators. Much of asthma drug development in the recent past had focused on new devices/formulations, new molecules in well-established classes, and new combination treatments, particularly of long-acting bronchodilators and corticosteroids. However, with the increasing knowledge of the underlying pathophysiology of the asthma phenotypes, new modes of addressing the inflammatory components of asthma are being developed based on specific phenotypes or endotypes. ${ }^{7}$ Examples of asthma phenotypes include early-onset versus late-onset, atopic versus non-atopic, or even more specific categories of asthma such as severe non-atopic asthma with frequent exacerbations. Examples of asthma endotypes include pathophysiologic criteria such as eosinophilic asthma, aspirin-exacerbated respiratory disease, or allergic bronchopulmonary aspergillosis. The subcategorization of asthma into phenotypes or endotypes is leading to a new era of precision medicine in asthma that will shape regulatory expectations and undoubtedly disrupt the traditional paradigms of treatment. Cleared drugs for asthma are shown in Table 1, and a brief description of their indications is in Table 2 .

\section{Asthma Drug Development by Class}

The development of asthma medications varies substantially by class and mechanism of action, reflecting the underlying pharmacology and therapeutic intent. For these reasons, the following asthma drug development discussion is broken out by class.

Short-Term Relievers/Short-Acting $\beta$ Agonists (SABAs). These agents are intended for as-needed use in addressing acute bronchospasm and wheezing, and their indication is not specific to a given disease, applying to either asthma or COPD. It is worth noting that most of the recent development in this class has focused on reformulations needed to complete the phase-out of chlorofluorocarbons as a part of international efforts to protect the ozone layer, rather than on new molecules. ${ }^{8}$ The most recent clearance of a novel SABA was for levalbuterol, the S-enantiomer of the traditional racemic albuterol. Despite the chlorofluorocarbon phase-out leading to a narrowing of options in SABA molecules (eg, pirbuterol is no longer available), no fully novel 


\section{Drug Development for Asthma And COPD}

Table 1. FDA Cleared Drugs for Asthma*

\begin{tabular}{|c|c|c|c|}
\hline \multicolumn{2}{|c|}{ CLASS } & Generic Name & Dosage Form \\
\hline$\beta_{2}$-adrenergic agonists & SABA & Albuterol sulfate & pMDI, DPI, inhalation solutions; oral tablets \\
\hline & & Levalbuterol tartrate & pMDI, inhalation solution \\
\hline & & Metaproterenol & Inhalation solution; oral syrup, tablets \\
\hline & LABA & Salmeterol & pMDI, DPI \\
\hline & & Formoterol & DPI, inhalation solution \\
\hline \multirow[t]{6}{*}{ Corticosteroids } & Inhaled & Beclomethasone propionate & pMDI \\
\hline & & Budesonide & DPI, inhalation suspension \\
\hline & & Ciclesonide & pMDI \\
\hline & & Fluticasone furoate & DPI \\
\hline & & Fluticasone propionate & pMDI, DPI \\
\hline & & Mometasone & DPI, pMDI \\
\hline Antimuscarinic agents & LAMA & Tiotropium & Soft mist inhaler \\
\hline \multirow[t]{4}{*}{ Combinations } & Corticosteroid/LABA & Fluticasone propionate/salmeterol & pMDI, DPI \\
\hline & & Budesonide/formoterol & pMDI \\
\hline & & Fluticasone furoate/vilanterol & DPI \\
\hline & & Mometasone/formoterol & DPI \\
\hline \multirow[t]{3}{*}{ Anti-leukotrienes } & Leukotriene synthesis inhibitor & Zileuton & Modified-release tablet \\
\hline & Leukotriene receptor antagonist & Montelukast & Tablets, granules \\
\hline & & Zafirlukast & Tablets \\
\hline \multirow[t]{3}{*}{ Biologic immune modulator } & Anti-IL5 antibody & Reslizumab & Intravenous infusion \\
\hline & & Mepoluzimab & Subcutaneous injection \\
\hline & Anti-IgE antibody & Omaluzimab & Subcutaneous injection \\
\hline $\begin{array}{l}\text { * Derived from the American Academy } \\
\text { FDA = Food and Drug Administration } \\
\text { SABA = short-acting } \beta_{2} \text {-adrenergic agc } \\
\text { LABA = long-acting } \beta_{2} \text {-adrenergic ago } \\
\text { pMDI = pressurized metered-dose inha } \\
\text { DPI = dry powder inhaler } \\
\text { LAMA = long-acting antimuscarinic ag }\end{array}$ & $\begin{array}{l}\text { f Allergy, Asthma, and Immunology web sit } \\
\text { sts } \\
\text { sts } \\
\text { ts }\end{array}$ & & \\
\hline
\end{tabular}

Table 2. Asthma Drugs and Indication Statements by Class*

\begin{tabular}{|c|c|}
\hline SABA & For the treatment or prevention of bronchospasm with reversible obstructive airways disease \\
\hline LABA (formoterol, salmeterol) & $\begin{array}{l}\text { For long-term, twice-daily administration as maintenance treatment of asthma and for prevention of } \\
\text { bronchospasm }\end{array}$ \\
\hline ICS & For maintenance treatment of asthma as prophylactic therapy \\
\hline LAMA (tiotropium) & For long-term, once-daily maintenance treatment of asthma \\
\hline Combination ICS/LABA & For the treatment of asthma \\
\hline Antileukotrienes & For prophylaxis and chronic treatment of asthma \\
\hline $\begin{array}{l}\text { Biologic immune modulator } \\
\text { (omalizumab) }\end{array}$ & $\begin{array}{l}\text { For the treatment of moderate-to-severe persistent asthma in patients with a positive skin test or in vitro reactivity } \\
\text { to a perennial aeroallergen and symptoms that are inadequately controlled with inhaled corticosteroids }\end{array}$ \\
\hline $\begin{array}{l}\text { Biologic immune modulators } \\
\text { (mepolizumab, reslizumab, } \\
\text { benralizumab) }\end{array}$ & For add-on maintenance treatment of patients with severe asthma with an eosinophilic phenotype \\
\hline $\begin{array}{l}\text { * Indications are broadly worded by class. } \\
\text { SABA = short-acting } \beta_{2} \text {-adrenergic agonists } \\
\text { LABA = long-acting } \beta_{2} \text {-adrenergic agonists } \\
\text { ICS = inhaled corticosteroids } \\
\text { LAMA = long-acting antimuscarinic agents }\end{array}$ & \\
\hline
\end{tabular}

molecule has been developed in this class in decades. Nonetheless, the paradigm for development is clear from the program for the chlorofluorocarbon reformulations and the levalbuterol program. ${ }^{9}$
For a new inhaled SABA molecule, the FDA's expectations would be similar to those for general drug development, which are laid out in the COPD Drug Development Overview, with the addition of juvenile animal studies 


\section{Drug Development for Asthma AND COPD}

(systemic and inhalational models) in the preclinical phase to account for likely use in the pediatric age group. Such pediatric considerations are generally waived for drug development targeted to treat COPD. Generating dose-ranging information in this class can be done relatively concisely, established through assessments of serial $\mathrm{FEV}_{1}$ measurements in stable subjects with asthma who have previously documented air-flow impairment with responsiveness to bronchodilators. Subjects with asthma are distinguished from those with COPD for these purposes (beyond a prior diagnosis) by a limited smoking history $(<10$ pack-year history), younger age (for clinical pharmacology studies, often 45 y or younger), and demonstrated response to SABA administration at screening of $\geq 12-15 \%$ improvement in $\mathrm{FEV}_{1}$ after 2 puffs of a cleared albuterol pMDI. Alternatively, for clinical pharmacologic dose-ranging, stable asthma subjects with relatively preserved air flow can be assessed with methacholine challenge testing prior to and then following the test drug for bronchoprotective properties.

Once an appropriate dose range is identified for a novel drug, FDA expectations would be for 2 or more phase 3 studies in a broader asthma population (typically targeting subject 12 y or older initially, including elderly asthmatics) to establish acute dosing effects on serial $\mathrm{FEV}_{1}$ (to determine maximum $\mathrm{FEV}_{1}$ response and duration of response or the $\mathrm{FEV}_{1}$ area under the curve above baseline) and subject responses to the drug at various time points within the trial, which are expected to be 12-16 weeks in duration. While SABAs are now used and labeled for asneeded use, these studies traditionally dosed subjects on a fixed schedule (eg, 4 times a day) to examine the efficacy and safety at what would result from a maximum dose. Such studies are usually placebo-controlled with rescue allowed with a cleared albuterol inhaler. Note that the FDA focuses on bronchodilation as the primary assessment in this setting. This is reflected in the labeled indications that refer to the treatment and prevention of bronchospasm. Prevention for SABAs specifically is supported either through studies to assess the mitigation of exerciseinduced bronchospasm (structured exercise testing in subjects with a documented history of exercise-induced airflow obstruction) or methacholine challenge testing.

In addition to the periodic serial $\mathrm{FEV}_{1}$ assessments, study subjects will generally assess daily morning and evening peak flows at home (using peak expiratory flow devices) and keep a symptom diary assessing traditional symptoms of interest, such as cough, shortness of breath (at rest or with exercise), nocturnal awakenings or wheezing. While the European Medicines Agency generally requires coprimary assessments of objective and subjective measures in asthma studies, the FDA has traditionally viewed $\mathrm{FEV}_{1}$ as the primary outcome for bronchodilators (SABAs, LABAs, and LAMAs) with symptom scores as informa- tive secondary assessments. Treatment failures and worsening asthma rates (eg, asthma exacerbations) would be important secondary end points as well, but in a 12-16week trial, these are unlikely to show a statistically important difference for SABAs. In addition to the replicate, shorter-term studies, the FDA requires a long-term safety study (usually with some measure of durable response included) for 12 months with at least 300 subjects examined for 6 months and at least 100 subjects examined for $1 \mathrm{y}$. These latter are minimums coming from an International Council for Harmonization document (http://www.ich.org/fileadmin/Public_Web_Site/ICH_ Products/Guidelines/Efficacy/E1/Step4/E1_Guideline.pdf, Accessed April 5, 2018.) for chronically dosed medications, and the actual numbers for a novel product frequently exceed these minimums. The long-term study can utilize as-needed dosing of the SABA, and such longer-term studies are often done through an open-label extension of one or both of the phase 3 studies. Particular attention is paid to exacerbation rates in comparison to any comparator control and the issues of paradoxical bronchospasm, wherein some characteristic of the formulation leads to further bronchoconstriction post-inhalation rather than relief. Such occurrences are particularly noteworthy after changes in formulation or inhaler components in a new program or after postclearance changes.

Long-Acting Bronchodilators. Unlike SABAs, the longer-acting bronchodilators are cleared not for relief of acute episodes on an as-needed basis, but as regularly dosed medications to prevent bronchospasm and ameliorate symptoms of air-flow obstruction. This changes the development pathway in comparison to SABAs.

LABAs. LABAs were first developed as stand-alone products, though asthma treatment guidelines and the development of combination products combining LABAs with corticosteroids have largely supplanted the use of single-ingredient LABA products in treating asthma. In addition, evidence of increases in serious asthma exacerbations has led to FDA warnings about the use of LABAs without concomitant ICS. Nonetheless, the development paradigm for LABAs as stand-alone products also informs some aspects of the combination products and therefore is worth reviewing.

Aside from the other expectations of standard drug development (in this case, also including toxicology in adults and juvenile animals), clinical pharmacology/dose-ranging with LABAs is similar to that of SABAs and is primarily based on effects of dose levels versus degree of bronchodilation as assessed by $\mathrm{FEV}_{1}$ responses, including metrics such as time to maximum effect, maximum $\mathrm{FEV}_{1}$ compared to pre-dose baseline, time until $\mathrm{FEV}_{1}$ returns toward baseline (eg, time until $\mathrm{FEV}_{1}$ returns to within $105-110 \%$ of pre-dose 


\section{Drug Development for Asthma AND COPD}

baseline), and the $\mathrm{FEV}_{1}$ area under the curve. At some point in the program, the sponsor must address the serial $\mathrm{FEV}_{1} /$ area under the curve both acutely (early after initiation of dosing) and chronically (weeks into a course of regularly administered medication) to assess for any tachyphylaxis with chronic administration. This can be a logistical challenge for a drug with a 12- or 24-h period of effect, as subjects may need to domiciled for extended periods. The characterization of acute bronchodilatory action can be easily accomplished in shorterterm phase 2 trials, while the documentation of any changes seen with chronic administration need to be considered as a part of the phase 3 program.

In contrast to the SABA paradigm, a LABA program utilizes regularly scheduled dosing throughout, including during long-term safety studies, because this is how these drugs are labeled. The end points for the phase 3 trials, as with SABAs, focus on spirometry and assessing changes in $\mathrm{FEV}_{1}$ over time both at the first dose and end of study. As with SABAs, the assessment of symptoms, peak expiratory flow, and other metrics of asthma control are followed as secondary end points. Unlike SABAs, an additional assessment with LABAs is the comparative need for rescue SABA use during the trial. For safety, attention is paid to exacerbation rates, particularly serious exacerbations (eg, requiring emergency department or hospital treatment) and death. Shortly after the initial marketing of salmeterol, the FDA began to receive reports of serious asthma deteriorations and deaths in patients treated with the new medication. This eventually led to the Salmeterol Multi-center Asthma Research Trial, ${ }^{10}$ which indeed identified an excess risk of such events. However, this effect was primarily seen in subjects not on concomitant ICS, and the absolute risk was small (although the relative risk was significant). This, along with some data from higherdose formoterol studies that were of concern to the FDA, ${ }^{11}$ led the FDA to require boxed warnings along with an expectation of post-clearance safety studies for new LABAcontaining products to examine rates of serious asthma events. ${ }^{12}$ GlaxoSmithKline, Merck, and AstraZeneca have all now reported reassuring findings that a LABA, when added to an ICS in a combination inhaler, does not lead to any signal for more serious adverse asthma events. ${ }^{13-16}$

LAMAs. To date, only one LAMA has been cleared for asthma as a maintenance, once-daily therapy: tiotropium administered by the proprietary inhaler (Respimat, Boehringer Ingelheim, Ingelheim, Germany). As this molecule was previously developed for COPD, the asthma program was not a full de novo program. Dose selection was obtained in short-term, crossover trials involving 5 doses of tiotropium. However, these studies did not yield definitive results that identified a single dose level. ${ }^{17}$ Therefore, 2 doses were included in the phase 3 trials $(2.5 \mathrm{mg}$ vs $5 \mathrm{mg}$ daily) and then compared to placebo and twice-daily sal- meterol. The primary end point for these trials was the mean change in $\mathrm{FEV}_{1}$ from pre-dose to peak $(0-3 \mathrm{~h})$, and the longer-term trials included a co-primary of change from baseline to trough (pre-dose) FEV 1 at the end of the study to document durability of response. Additional end points included assessments of peak expiratory flows (morning and evening), asthma exacerbations, asthma control, and health-related quality of life outcomes assessed by the Asthma Quality of Life Questionnaire instrument. The drug was cleared primarily on the basis of the bronchodilator effects documented in multiple phase 3 studies. ${ }^{18}$ Interestingly, these studies showed a decrease in asthma exacerbations sufficient for mention in the labeling, but not a specific indication statement. It is notable that, unlike the LABAs, there was no FDA requirement for a post-clearance safety study. ${ }^{19}$ In summary, the development of a LAMA in asthma appears to be quite similar to what might be required for a new LABA, however, data to date would not suggest a risk of rare, serious asthma exacerbations occurring with LAMAs as single agents. Therefore, no boxed warning is currently required by the FDA, and no requirement for a large outcomes trial appears to be likely for any subsequent LAMA developed for asthma.

Anti-Inflammatory Medications. These medications are aimed at affecting the underlying airway inflammation, rather than having a direct action on airway smooth muscle and acute bronchospasm. As such, the development pathway focuses on assessments of clinical end points reflective of longer-term asthma control over acute bronchodilator effects.

ICS. While systemic steroids have long been used in the setting of asthma exacerbations or severe, hard to control disease, the use of ICS has become a mainstay of therapy for asthma due to their broad effectiveness and relative safety.

In developing a new corticosteroid for inhalation, one key consideration is the oral or gastrointestinal (GI) tract bioavailability. With most inhalation devices, a substantial portion of a dose emitted from a device impacts in the mouth and pharynx and then is swallowed. High oral bioavailability can therefore negate some of the safety advantages of the inhaled route. While the lung is also a source of systemic exposure for corticosteroids, for those moieties with a limited GI bioavailability, the total bioavailability may be well below $5 \%$, but for a moiety like beclomethasone, which has a relatively high fractional GI absorption (of the parent and active metabolite), the bioavailability of an inhaled dose may be well in excess of $50 \%$. This has implications for issues such as growth inhibitory effects in children and long-term corticosteroid toxicity on bone, eyes, and other target organs. Most newer ICS exhibit very low to negligible oral bioavailability.

While much work has been conducted to define acute and sensitive biomarkers that could provide definitive, 


\section{Drug Development for Asthma AND COPD}

short-term assessments of dose versus response for ICS, to date few such assessments have been shown to be reliable and valid. Assessments such as the fraction of exhaled nitric oxide $\left(\mathrm{F}_{\mathrm{ENO}}\right)$ hold promise. ${ }^{20} \mathrm{~F}_{\mathrm{ENO}}$ may be useful for a sponsor to assess exposure-response in early trials to define doses to be taken into phase 2 and phase 3 studies; however, the FDA has been less receptive of $\mathrm{F}_{\mathrm{ENO}}$ as definitive data in identifying the appropriate clinical dose(s) to the degree of obviating phase 2 trials. Without an acute, sensitive clinical or biomarker measure to define doseeffect, dose ranges to be examined would need to be initially extrapolated from in vitro binding studies and animal models, but the FDA requires phase 2 studies of considerable size and length (eg, 8-12 weeks) to provide data to inform the appropriate, limited range of doses to take into phase 3 . The end points used in the phase 2 trials are often the same as those in phase 3 trials, with a focus on improvement of trough $\mathrm{FEV}_{1}\left(\mathrm{FEV}_{1}\right.$ assessed prior to the first daily dose of the study drug and before any bronchodilator) over time compared to baseline, as well as symptom scores (eg, breathlessness, cough, wheeze, and nocturnal awakenings) and bronchial hyperreactivity as assessed by methacholine or similar challenges studies.

For phase 3 studies, the FDA expects a range of asthma severity to be addressed in the development program (mild, moderate, severe with or without dependence on oral steroids). Additionally, the patient population has usually included adults and adolescents (eg, subjects $12 \mathrm{y}$ and older). While pediatric studies would eventually be required for an asthma drug, these studies may be deferred until safety and effectiveness is established in adolescents and adults. Nonetheless, examining a range of asthma severity results in the need to conduct multiple studies with differing designs. However, it is common regulatory practice for this suite of multiple phase 3 studies to be considered as replication of effectiveness if all of the studies are positive, rather than replication of each specific dose/population. In general, these studies individually examine more than 1 dose level, but the dose levels themselves may vary between studies. For instance, if the phase 2 trials have shown a range for the experimental moiety that might be considered as low, medium, and high dose, the low and moderate doses might be examined in trials targeting a relatively milder asthma population (eg, those previously not on ICS), while moderate and high doses might be evaluated for those patients not adequately controlled on lower-dose ICS at study entry or in patients with more severe asthma.

The FDA's preferred primary end point in phase 3 ICS studies is typically an objective measure of air flow (trough $\mathrm{FEV}_{1}$ ) with secondary assessments of asthma symptoms (with or without formal asthma control, peak expiratory flow, and exacerbation rates). The FDA has accepted less validated but historically used measures of asthma symptoms and control, but has also more recently allowed the use of specifically developed instruments like the Asthma Control Test and the Asthma Quality of Life Questionnaire. For subjects with milder cases, placebo-controls are acceptable and generally encouraged, while placebo-controls may also be possible for subjects with moderate asthma if well-defined destabilization criteria are in place so that any subject whose asthma deteriorates to a predefined degree is rescued and removed from the study. ${ }^{21}$ In this instance, the rate of subjects meeting these criteria can itself be considered an end point, with effective doses showing a reduced treatment-failure rate. Safety assessments in these studies would be fairly routine, but they should include a focus on issues and rates of common or possible adverse effects of ICS, including oral candidiasis, hoarseness, upper and lower respiratory tract infections, and proximate bronchospasm.

The safety program for a novel ICS should examine issues of systemic exposure at higher or maximum chronic doses, such as hypothalamic pituitary axis effects, ocular safety (eg, cataract formation), and bone density. These can usually be addressed within phase 3 efficacy studies and the long-term safety assessments as sub-studies without necessarily requiring specific designs. After the observation that intranasal beclomethasone could lead to growth effects in younger children, the FDA instituted a requirement to address growth effects for new ICS products and has issued guidance on the specific conduct of these studies. ${ }^{22}$

Anti-leukotrienes. Novel drug development in this class appears quiescent. However, as oral drugs with actions beyond the airway, the preclinical and early clinical work would be largely traditional. Dose finding would start with in vitro data to define likely exposure ranges, and animal data/models would be used to validate both the actions (proof of principle of the pharmacology) and further refinement of clinical exposure targets/dosing. Interestingly, despite the role of leukotrienes in the manifestations of allergic reactions, defining asthma phenotypes that might be particularly responsive to this class of medication has not been fruitful. ${ }^{23}$ The clinical trials for these agents are typical for a chronically administered asthma-controller paradigm, focusing on pre-dose $\mathrm{FEV}_{1}$ improvements over time (compared to placebo in addition to standard care), with secondary assessments of peak expiratory flow and asthma symptoms, including nocturnal awakenings and change in use of $\beta$ agonist relievers. As with other asthma medication development programs, these trials should first be done in adults/adolescents before being extended into younger asthma patients (down to age $2 \mathrm{y}$ ). It is worth noting that, for the youngest children, based on similarity of disease and expected response, the FDA has allowed the use of PK data, supplemented with demonstrated safety and non-inferential efficacy evaluations, to inform clearance (see montelukast labeling ${ }^{24}$ ). 


\section{Drug Development for Asthma AND COPD}

Combination ICS/LABA Therapy. A number of combinations of LABA with ICS have been developed for asthma. In part, this solves a concern over the safety of LABAs in asthma without concomitant corticosteroids and provides for enhanced convenience as well as a potential positive perceptible benefit (relief of bronchospasm) of the combination that a patient would not perceive with a single ingredient ICS. ${ }^{25}$

In many ways, the development of these combinations are informed by the FDA's expectations for the development of the components, but with the complexity of having to address the efficacy of the combination within a given study with end points reflective of the differing pharmacology/pharmacodynamics. Dose finding for the combination of 2 previously cleared moieties will heavily depend on the known dosing information for each component. Consideration would have to be given to any potential pharmacologic interactions between the 2 drugs, however, and the pharmaceutics of the delivery device (the inhaler and its delivery characteristics, including particle sizing of each ingredient) will also factor into targeted dosing. These considerations of the potential effects of changes in the delivery device and pharmaceutics are important to discuss with the FDA during development, but because they are circumstantial in nature, no general advice is warranted. For combination products in which one or more of the ingredients are novel, separate dose-ranging data for each moiety, delivered via an inhaler as close to the proposed combination device as possible, is advisable. These studies are discussed in the sections about ICS and LABAs.

A combination product invokes an FDA regulatory requirement (21 CFR 300.50) that generally requires the developer to demonstrate that a population exists who would be served by the combination, and that each component of the combination contributes to the clinical effect of the combination product. For combinations where each component is expected to contribute to efficacy, this generally will mean a factorial study design (that is, component A vs $\mathrm{B}$ vs $\mathrm{AB}$ with and without placebo-control). In practice, a full factorial design would mean a study of 12-16 weeks in duration that targets an asthma population of relevant severity to examine the ICS at an appropriate, previously identified dose versus the LABA at a defined dose compared to the combination of the 2 components combined at the same nominal doses. To avoid the pharmaceutics issue, these 3 doses should all be delivered by the same delivery devices and formulations (with the exception of the actives) supported by in vitro characterization to show that the expected lung delivery is not affected by the changes in the active moieties. Because the use of LABAs without concomitant corticosteroids has raised safety concerns regarding asthma deaths, it is possible that one arm can be left out of the factorial considerations, so that the treatment arms are placebo versus ICS versus LABA/ICS, where the comparison of the LABA/ICS to the ICS alone and to the placebo will allow one to assess the added effect of the LABA.

Primary end points for such factorial studies in asthma are complex, as the appropriate clinical measures of ICS effects are distinct from those of LABAs. Co-primary end points that reflect both attributes are necessary, such as pre-dose $\mathrm{FEV}_{1}$ over time and at the end of the study is usual for the ICS component, and timed $\mathrm{FEV}_{1}$ (peak, area under the curve, duration) after dosing is usual for the LABA at first dose, periodically during the study, and at the end of study. Other supportive asthma end points also should be assessed (eg, symptom scores, asthma control, exacerbation rates, and morning and evening peak expiratory flows) and are relevant to both agents. As with any ICS program, a number of studies may be needed to assess patients requiring differing doses of the ICS due to their underlying asthma severity (ie, higher doses for greater severity).

Safety assessments and end points are informed by the safety concerns of the population (asthma) and the underlying pharmacologic class issues described above. Notably, issues of adverse LABA effects on serious asthma events or death and adverse ICS effects on growth, bone, hypothalamic pituitary axis, or ophthalmologic effects may be invoked by the FDA, especially if one or more of the moieties is novel.

Longer-term safety and effectiveness studies would not necessarily have to be factorial in design if use of a given combination has already been substantiated and the regulation therefore has been addressed. For instance, an examination of the effect of a LABA/ICS combination inhaler in mitigating serious asthma exacerbations versus placebo (both added to standard care) may be acceptable given the feasibility of doing a factorial study in such a situation if the combination rule has otherwise been addressed.

Anti-IgE and Anti-IL-5 Therapies. The development of biologic therapies for asthma has been in process for many years, but success in clinical development has been challenging, in part reflecting the incomplete knowledge of the roles of various specific, isolated targets in asthma overall or even within specific clinical phenotypes. Nonetheless, such agents have been cleared, and they are offering new therapeutic options for appropriate patients as well as new insights into the pathophysiology of asthma.

The first of the biologics cleared by the FDA for asthma was the humanized, anti-immunoglobulin E (IgE), known as omaluzimab. This drug, which targets a major contributor in the complex chain leading to allergic responses (ie, the $\operatorname{IgE}$ molecule), was developed for allergic asthma as the targeted phenotypic population. However, defining uniquely responsive patients was challenging, and ulti- 


\section{Drug Development for Asthma AND COPD}

mately the drug was studied in subjects with asthma and a positive, clinically relevant allergen sensitivity (eg, by skin testing or radioallergosorbent testing). While much of the use of this agent has focused on severe allergic asthma, the clinical studies were done with subjects who had what could be considered moderate asthma, as they were only required to have chronic asthma (for at least $1 \mathrm{y}$ ) and to be receiving treatment with ICS, in addition to the demonstration of a positive skin test to at least one perennial aeroallergen and an elevated total serum IgE. ${ }^{26}$ In the 4 pivotal trials, the design focused on being able to wean the use of ICS (which was shown in all 4 trials) and to improve exacerbation rates and other measures of asthma control, including spirometry. There was, in 3 of the 4 trials, a demonstrated reduction in exacerbation rate compared to placebo, in addition to reductions in ICS use. However, in the original trial examining the most severe patient population, no significant effect on exacerbation rate was seen. Furthermore, there were inconsistent results on end points such as $\mathrm{FEV}_{1}$. Clinically, this leads to a challenge in defining individual treatment response given that asthma is a variable disease and traditional methods of assessing objective response (eg, lung function test) were not necessarily predictive. Testing for baseline IgE level in patients was deemed important primarily to identify dosing intensity rather than overall likelihood of response. The evolution of the clinical use of omalizumab in allergic asthma has been driven as much by post-clearance clinical experience as by regulatory refinement of the labeling.

More recently, other monoclonal antibodies have been in development for asthma, mainly targeting pathways leading to enhanced Type 2 T-helper (Th2) immune response. These targets have included interleukins (eg, IL-4, IL-5, IL-13), along with other elements of the Th2 response, such as anti-thymic stromal lymphopoietin (anti-TSPL). ${ }^{27}$ The most advanced of these therapies are the anti-IL-5 antibodies (ie, mepolizumab, reslizumab, and benralizumab), but these moieties have an interesting and instructive path to regulatory clearance. While IL-5 as a driver of the allergic/Th2 response was well established, demonstrating a clinical benefit of these agents was a development challenge. ${ }^{28}$ Early phase 2 studies focusing on a broad phenotype of persistent asthma were disappointing, providing very little evidence of tangible clinical benefit. However, a small study $(N=20)$ that examined the use of mepolizumab in subjects on prednisone with identified elevations in sputum eosinophils showed encouraging results in exacerbation rates, despite the small sample size. ${ }^{29}$ Unfortunately, sputum eosinophilia is not readily assessed in most clinical settings. Eventually, the phase 3 trials for both mepolizumab and reslizumab were conducted in subjects with evidence of important elevations of blood eosinophils (as a correlate to airway eosinophilia) and histories of frequent exacerbations requiring systemic corticosteroids despite moderate- to high-dose ICS. ${ }^{30,31}$ However, the specific entry criterion for the level of serum eosinophils differed in the 2 programs. Both drugs were shown to reduce subsequent exacerbation rates by approximately $50 \%$ in subjects with moderately severe asthma identified by elevated serum eosinophils, with more modest and variable results on traditional end points such as $\mathrm{FEV}_{1}$ change over time or asthma symptom scores. Benralizumab is the most recently cleared anti-IL-5 antibody and it also showed similar reductions of approximately $50 \%$ in exacerbations rates. The FDA ultimately cleared all 3 anti-IL-5 agents as add-on maintenance therapy for patients with severe asthma and an eosinophilic phenotype, without further specifying in the indication statement what the clinical criterion should be for that phenotype.

The lessons for other targeted biologics from the development of the IL-5 antibodies are clear: Rather than targeting a broad population of asthmatics, the program should focus on identifying feasible and appropriate biomarkers that enhance the likelihood of response to the therapy and that the likely outcome of interest will be asthma control/exacerbation rates, rather than the traditional controller medication assessment of improvement in trough $\mathrm{FEV}_{1}$ over time. Because trials to demonstrate improvements in exacerbation rates are lengthy and large (given historic precedent and likely effect sizes), this means substantial work must be done in early development to identify the best biomarkers to use as a means for enriching or even restricting entry into the phase 3 studies. This is a daunting challenge, however, as a recent set of studies reported for a number of other agents targeting the Th2 response (eg, anti-IL13) have not shown a clear predictive biomarker associated with a substantial clinical effect. ${ }^{32}$

\section{COPD Drug Development Overview}

The FDA has published 2 draft guidances with respect to the clinical development of drugs for COPD: one in November 2007, and one in May 2016. The 2016 guidance is similar to the 2007 guidance, with the addition of an appendix discussing the use of the St George Respiratory Questionnaire (SGRQ) in COPD studies. The 2016 Guidance for Industry, entitled Chronic Obstructive Pulmonary Disease: Developing Drugs for Treatment, ${ }^{33}$ is available at the FDA Website, and the authors recommend this guidance to interested readers for further understanding of the FDA's framework for COPD drug development.

The guidance opines that there is a pressing need to develop new drugs for COPD, particularly ones that may affect disease progression, because currently available therapies are mostly for symptomatic treatment. Potential drugs for COPD are categorized broadly by the FDA as improving air flow obstruction; providing symptom relief (eg, cough or dyspnea); modifying or preventing exacerbations; 
Drug Development for Asthma And COPD

Table 3. Drugs Cleared by the FDA for COPD

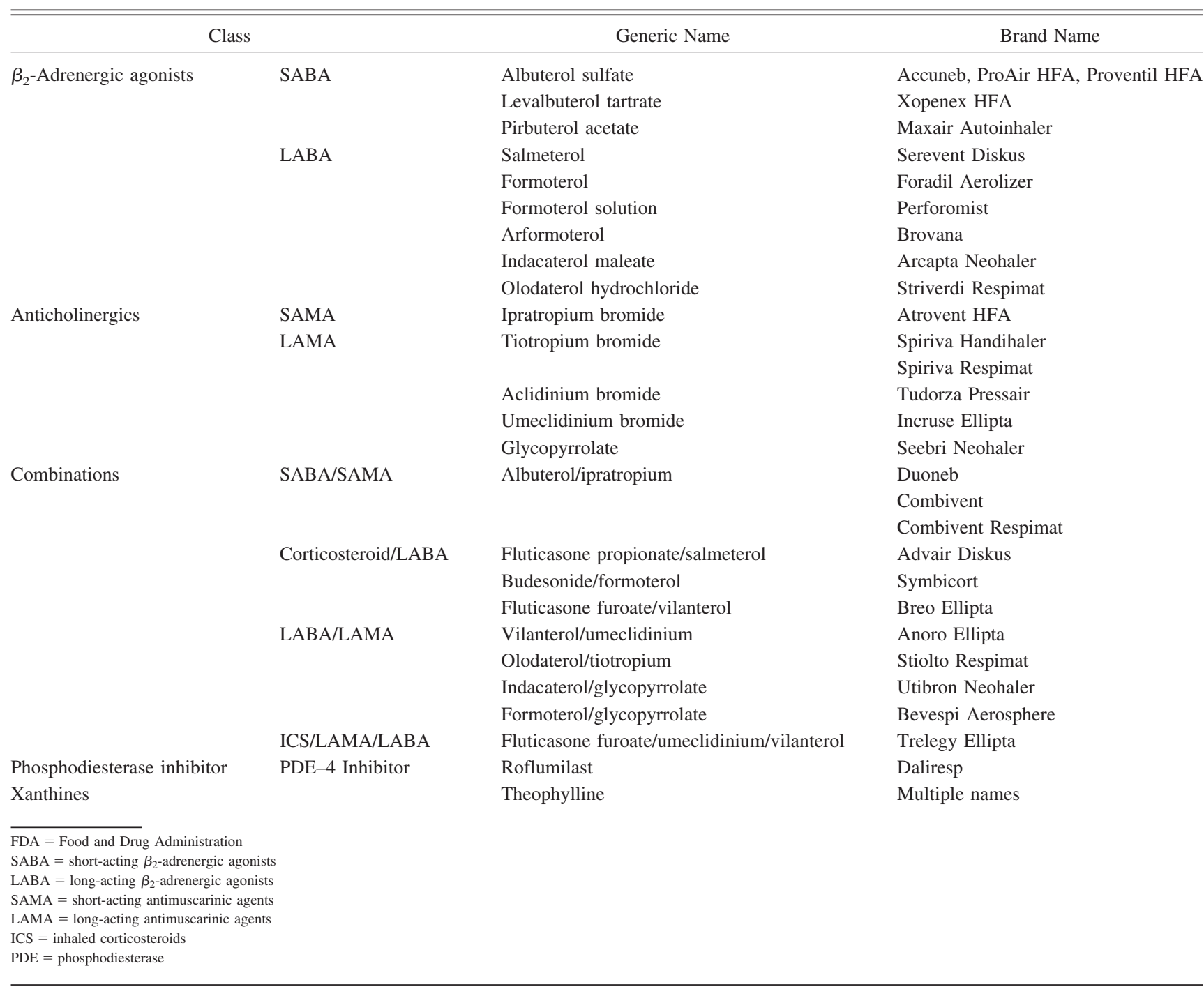

altering disease progression; and modifying lung structure. Cleared drugs for COPD and their labeled indications, including those that have SGRQ findings described in the label, are shown in Tables 3 and 4 .

As with asthma, a critical challenge for inhaled drugs for COPD is to determine the effective dose(s) and regimen. Phase 2 dose-finding studies often use $\mathrm{FEV}_{1}$ as a sensitive primary efficacy end point; however, surrogate biomarker end points or even trends on clinical end points can be evaluated to help further elucidate the dose-response. For some COPD indications, such as preventing exacerbations, the current state of biomarkers and physiological understanding are such that it may be difficult to perform highly informative phase 2 dose-finding trials if the investigational drug demonstrates no bronchodilatory effects. In such cases, phase 3 trials may need to include at least 2 doses of drug versus placebo as a means of pro- viding sufficient dose-response information. Typically, most COPD drugs are cleared at a single dose/regimen. To support multiple dose options of a drug for COPD, a sound rationale based on clinical data must be provided. Absent this, the FDA has cautioned in labeling that higher doses of a given drug showed no additional benefit. Advair Diskus, for example, is cleared only at the 250/50 strength for COPD, with labeling noting that an efficacy advantage of the higher-strength Advair 500/50 had not been demonstrated. ${ }^{34}$

Generally, 2 confirmatory phase 3 trials are needed to support clearance but the 2 trials do not need to be identical. For example, a first pivotal COPD trial might be designed to enroll subjects with moderate COPD, while a second might enroll subjects with more severe COPD. A single long-duration trial may be sufficient for a drug claiming to alter COPD disease progression, modify lung struc- 


\section{Drug Development for Asthma And COPD}

Table 4. COPD Drugs, Their Indications, and SGRQ Findings in Labeling*

\begin{tabular}{|c|c|c|}
\hline Drug Class/Product & Indication & $\begin{array}{l}\text { SGRQ } \\
\text { in Label }\end{array}$ \\
\hline \multicolumn{3}{|l|}{ SABA Therapy } \\
\hline Albuterol & For the treatment or prevention of bronchospasm with reversible obstructive airway disease & No \\
\hline Levalbuterol & For the treatment or prevention of bronchospasm with reversible obstructive airway disease & No \\
\hline Pirbuterol & For the treatment or prevention of bronchospasm with reversible obstructive airway disease & No \\
\hline \multicolumn{3}{|l|}{ SAMA Therapy } \\
\hline Ipratropium bromide & $\begin{array}{l}\text { For the maintenance treatment of bronchospasm associated with COPD, including chronic } \\
\text { bronchitis and emphysema }\end{array}$ & No \\
\hline \multicolumn{3}{|l|}{ SABA/SAMA Therapy } \\
\hline Albuterol sulfate/ipratropium bromide & $\begin{array}{l}\text { For patients with COPD on a regular aerosol bronchodilator who continue to have } \\
\text { bronchospasm and require a second bronchodilator }\end{array}$ & No \\
\hline \multicolumn{3}{|l|}{ LABA Therapy } \\
\hline Salmeterol & $\begin{array}{l}\text { For long-term maintenance treatment of bronchospasm associated with COPD (including } \\
\text { emphysema and chronic bronchitis) }\end{array}$ & No \\
\hline Formoterol & $\begin{array}{l}\text { For long-term maintenance treatment of bronchospasm associated with COPD (including } \\
\text { emphysema and chronic bronchitis) }\end{array}$ & No \\
\hline Arformoterol & $\begin{array}{l}\text { For long-term maintenance treatment of bronchospasm associated with COPD (including } \\
\text { emphysema and chronic bronchitis) }\end{array}$ & No \\
\hline Indacaterol & $\begin{array}{l}\text { For long-term maintenance treatment of bronchospasm associated with COPD (including } \\
\text { emphysema and chronic bronchitis) }\end{array}$ & Yes \\
\hline Olodaterol & $\begin{array}{l}\text { For long-term maintenance treatment of bronchospasm associated with COPD (including } \\
\text { emphysema and chronic bronchitis) }\end{array}$ & No \\
\hline \multicolumn{3}{|l|}{ LAMA Therapy } \\
\hline $\begin{array}{l}\text { Tiotropium (Spiriva Handihaler and } \\
\text { Respimat) }\end{array}$ & $\begin{array}{l}\text { For long-term maintenance treatment of bronchospasm associated with COPD (including } \\
\text { emphysema and chronic bronchitis) and for reducing COPD exacerbations }\end{array}$ & No \\
\hline Aclidinium & $\begin{array}{l}\text { For long-term maintenance treatment of bronchospasm associated with COPD (including } \\
\text { emphysema and chronic bronchitis) }\end{array}$ & No \\
\hline Umeclidinium & $\begin{array}{l}\text { For long-term maintenance treatment of bronchospasm associated with COPD (including } \\
\text { emphysema and chronic bronchitis) }\end{array}$ & Yes \\
\hline Glycopyrrolate & $\begin{array}{l}\text { For long-term maintenance treatment of bronchospasm associated with COPD (including } \\
\text { emphysema and chronic bronchitis) }\end{array}$ & Yes \\
\hline \multicolumn{3}{|l|}{ ICS/LABA Therapy } \\
\hline Fluticasone propionate/salmeterol & For maintenance treatment of airflow obstruction and reducing exacerbations & No \\
\hline Budesonide/formoterol & For maintenance treatment of airflow obstruction and reducing exacerbations & Yes \\
\hline Fluticasone furoate/vilanterol & For maintenance treatment of airflow obstruction and reducing exacerbations & Yes \\
\hline \multicolumn{3}{|l|}{ LABA/LAMA Therapy } \\
\hline Vilanterol/umeclidinium & Long term maintenance treatment of airflow obstruction & No \\
\hline Olodaterol/tiotropium & Long term maintenance treatment of airflow obstruction & Yes \\
\hline Indacaterol/glycopyrrolate & Long term maintenance treatment of airflow obstruction & Yes \\
\hline Formoterol/glycopyrrolate & Long term maintenance treatment of airflow obstruction & Yes \\
\hline \multicolumn{3}{|l|}{ ICS/LAMA/LABA Therapy } \\
\hline Fluticasone furoate/umeclidinium/vilanterol & $\begin{array}{l}\text { For patients on Breo Ellipta who may benefit from extra bronchodilation or for patients } \\
\text { already on Breo Ellipta and Incruse Ellipta }\end{array}$ & Yes \\
\hline \multicolumn{3}{|l|}{ Phosphodiesterase inhibitor therapy } \\
\hline Roflumilast & $\begin{array}{l}\text { Reduces the risk of COPD exacerbations in patients with severe COPD associated with } \\
\text { chronic bronchitis and a history of exacerbations }\end{array}$ & No \\
\hline \multicolumn{3}{|l|}{ Xanthines } \\
\hline Theophylline & $\begin{array}{l}\text { Treatment of symptoms and reversible airflow obstruction associated with asthma and } \\
\text { other chronic lung diseases (emphysema and chronic bronchitis) }\end{array}$ & No \\
\hline $\begin{array}{l}\text { * Indications are not precisely worded, but are generally consi } \\
\text { SGRQ }=\text { St George Respiratory Questionnaire } \\
\text { SABA }=\text { short-acting } \beta_{2} \text {-adrenergic agonists } \\
\text { SAMA }=\text { short-acting antimuscarinic agents } \\
\text { LABA }=\text { long-acting } \beta_{2} \text {-adrenergic agonists } \\
\text { LAMA = long-acting antimuscarinic agents } \\
\text { ICS = inhaled corticosteroids }\end{array}$ & stent with labeling. Generally consistent with labeling as per the FDA website viewed on March 25, 2018. & \\
\hline
\end{tabular}




\section{Drug Development for Asthma AND COPD}

ture, or modify/decrease exacerbation events, as these are very challenging claims. Any single trial would need to be very well designed and carefully conducted, and it would need to show robust and convincing results.

The duration of pivotal COPD studies are at least 3 months for a bronchodilator drug that uses improved $\mathrm{FEV}_{1}$ as its primary end point, and 6 months for a nonbronchodilator drug that uses $\mathrm{FEV}_{1}$ as its primary end point. For symptom relief, the duration of pivotal studies is at least 6 months; for an exacerbation claim, the durations are at least $1 \mathrm{y}$. These durations are partly driven by the need to allow sufficient time to observe enough events. For a drug that seeks a claim to alter disease progression, a trial of at least $3 \mathrm{y}$ is needed to demonstrate a change in the slope of $\mathrm{FEV}_{1}$ fall. For drugs that seek to modify lung structure, a trial would generally need to be several years in duration due to the slow pace of lung destruction in COPD. While asthma therapies generally need to be developed for pediatrics as well as adults, COPD therapies are generally developed only for use in adults and are therefore exempt from pediatric development requirements.

As with asthma, biomarkers are of interest in determining COPD phenotypes and for use in refining clinical trial enrollment criteria. These may also be useful in phase 2 trials if they are sensitive to dose-response. The COPD Biomarker Qualification Consortium was initiated in 2010 by the COPD Foundation with encouragement from the FDA and the National Heart, Lung, and Blood Institute to develop a biomarker qualification process for COPD. ${ }^{35}$ The first such biomarker, cleared by the COPD Biomarker Qualification Consortium in 2015, was plasma fibrinogen, which was found to be predictive of mortality and hospitalized exacerbations in patients with COPD. ${ }^{36}$ Clinical validation for this biomarker as a marker of higher exacerbation risk was largely based on the Evaluation of COPD Longitudinally to Identify Predictive Surrogate End Points (ECLIPSE) study, ${ }^{37}$ but this was bolstered by other studies. Findings showed that high fibrinogen levels $(>350 \mathrm{mg} / \mathrm{dL}$ ) were present at baseline in $44.7 \%$ of individuals with COPD, and these subjects had an increased risk of COPD exacerbations within 12 months (hazard ratio $1.64,95 \%$ CI 1.39-1.93). ${ }^{36}$ An FDA guidance for industry provides the context of use in interventional clinical trials of subjects with COPD, noting that fibrinogen levels could be used as an enrichment factor in COPD exacerbation trials but not currently as an end point. ${ }^{38}$ The use of any enrichment approach in identifying subjects for pivotal exacerbation trials, however, may also affect labeling and lead to a more restricted indication.

Primary end points for cleared COPD drugs have generally focused on pulmonary function or COPD exacerbations. For drugs that aim to treat air-flow obstruction (ie, bronchodilators), $\mathrm{FEV}_{1}$ is the primary efficacy end point and is considered a direct measure of the drug's benefit.
For non-bronchodilator drugs, $\mathrm{FEV}_{1}$ can also be a primary efficacy outcome, but in this case $\mathrm{FEV}_{1}$ is more of a surrogate assessment, and efficacy should also be supported by co-primary or secondary clinical efficacy outcomes, such as symptoms or patient-reported outcomes. For bronchodilators, the FDA's expectation is that the full FEV characterization, including peak, trough, and every time point in between, will show robust separation from placebo. The FDA does not endorse a level of trough-FEV ${ }_{1}$ benefit that is known to be clinically meaningful. A $100 \mathrm{~mL}$ change from baseline for drug versus placebo has been recognized in the literature as being perceived by patients, correlating with fewer relapses following exacerbations and falling in the range for the minimal mean trough effect met by all cleared bronchodilators for COPD. ${ }^{39}$

A reduction in moderate to severe COPD exacerbations (requiring systemic corticosteroid intervention or hospitalization) has also been a primary efficacy outcome in COPD trials, with drugs that currently carry this indication showing an approximately $20-30 \%$ relative reduction in risk. While COPD exacerbations are often defined by treatment intervention (eg, the need for oral steroids or antibiotics), it is important to also collect clinical information on the case report to confirm that the intervention was generally needed and not simply implemented without due cause. Because inhaled steroids in COPD have been associated with pneumonia risk, exacerbation studies within this class must clearly define exacerbations versus pneumonia.

Additional end points aside from $\mathrm{FEV}_{1}$ or COPD exacerbations that may be considered include other pulmonary function test findings (FVC or residual volume (RV)/total lung capacity (TLC) can be considered as a reflection of hyperinflation). While there is no precedence for use of this ratio as a primary end point in COPD, elevations of RV/TLC reflect air trapping and may be a relevant end point in COPD. Improvements in FVC in COPD as a primary end point also have no regulatory precedence, but FVC has been used as primary outcome in trials for idiopathic pulmonary fibrosis. If considered for a COPD trial, RV/TLC and FVC should be discussed in advance with regulatory authorities, with consideration given as to why $\mathrm{FEV}_{1}$, the more traditionally accepted end point, is not being proposed. Exercise capacity, as assessed by the standardized 6-min walk test or other standardized assessments, such as treadmill or cycle ergometry, can be considered, but this end point has high inter- and intrapatient variability, which has implications on assay sensitivity and power. Therefore, unless a dramatic effect on exercise capacity is anticipated, this outcome would generally be considered as a supportive outcome measurement.

Symptoms of COPD (eg, cough, dyspnea) are key manifestations of COPD for patients and yet are generally considered as secondary outcomes due to their subjective nature and the lack of precision and standardization in 
measurement. Specifically, the FDA's COPD Guidance notes that the Medical Research Council dyspnea score, the Borg Scale, and the Mahler Baseline Dyspnea Index/Traditional Dyspnea Index are not acceptable as primary outcome variables, nor can they be used to support specific labeling claims. Health-related quality of life instruments, including the SGRQ, the SGRQ-COPD (a COPD-specific version of the SGRQ), and the Chronic Respiratory Questionnaire are not considered appropriate as solitary primary end points by the FDA. ${ }^{40-42}$ However, both the SGRQ and the SGRQ-C are acceptable for use in COPD trials, and positive results may be included in labeling as per the FDA's 2016 COPD Guidance. ${ }^{33}$ The SGRQ can be a coprimary or key secondary end point that establishes clinical benefit of a drug for COPD, but it is not recommended by the FDA as a sole primary end point.

Additional guidance on patient-reported outcomes for COPD may derive from the FDA's Compendium of Clinical Outcomes Assessments, which lists various tests that have been previously used in clinical trials. ${ }^{43}$ The FDA created the list of clinical outcome assessments based on a review of labeling for new molecular entities cleared from 2003 to 2014. The Compendium is intended to promote patient-focused drug development. If an outcome measure is on this list, it is not a definitive indication that the measure is appropriate as a primary outcome, and it does not necessarily equate to FDA endorsement. Nonetheless, if an outcome measure is listed, it may be a viable end point to discuss with the FDA to support labeling claims. For COPD, end points listed in this compendium include lung function $\left(\mathrm{FEV}_{1}\right)$, the SGRQ, patient diaries, and the EXACT-PRO instruments. The EXACT-PRO instruments include the EXACT (EXAcerbations of Chronic Pulmonary Disease Tool), which is a 14-item patient-reported outcome daily diary used to quantify and measure exacerbations of COPD, and the E-RS (Evaluating Respiratory Symptoms), which is an instrument designed to follow respiratory symptoms in stable COPD patients. ${ }^{44}$ The E-RS utilizes 11 of the respiratory symptoms within EXACT. Both EXACT and the E-RS are useful tools to consider including in phase 2 or phase 3 COPD trials to better understand patient outcomes and to possibly inform dose selection.

\section{COPD Drug Development by Class}

\author{
Short-Term Relievers/Short-Acting $\beta$ Agonists \\ (SABAs) or Short-Acting Muscarinic Antagonists \\ (SAMAs). Cleared SABAs include albuterol sulfate, \\ levalbuterol titrate, and pirbuterol acetate (no longer \\ marketed). SAMAs include ipratropium bromide, which \\ is available either alone or in combination with albu- \\ terol sulfate (SABA/SAMA).
}

Indication statements vary, depending on the agent, and imply either a short-term rescue use for relief of bronchospasm or a maintenance use specific to COPD:

- SABAs are cleared for relief of bronchospasm, with most products not specifying asthma or COPD in the indication.

- SAMAs are indicated for the maintenance treatment of bronchospasm associated with COPD, including chronic bronchitis and emphysema.

- The combination of albuterol sulfate/ipratropium bromide is indicated for patients with COPD on a regular aerosol bronchodilator who continue to have evidence of bronchospasm and who require a second bronchodilator.

There is no formal definition for a short-acting bronchodilator, but their duration of action is known to be generally 4-6 h. The development of SABAs has been covered in the Asthma Drug Development by Class section. The development of SAMAs is generally similar, although these drugs have generally been developed for patients with COPD, not asthma.

Combination drugs (such as a SAMA/SABA) are challenging and submissions must show the added benefit of each component to bronchodilation as per the FDA combination rule. A full understanding of each single agent's benefit as a bronchodilator is required, even if the single agents are not being considered for market. Full characterization of the mono-components is expected, with robust dose-ranging studies that use the same devices as that for the combination. PK data also need to substantiate the lack of pharmaceutical interaction for the combination versus the single ingredients. When a full understanding of the single ingredients is achieved and the lack of pharmaceutical interactions is established, traditional phase 3 factorial design trials can be done to establish efficacy for the combination versus the mono-components. Such trials are generally placebo-controlled to fully understand the safety profile of the combination.

Safety concerns for SABAs include hypokalemia, hyperglycemia, and tachycardia. Safety concerns for SAMAs include dry mouth, urinary retention, and cardiac arrhythmias. These must be adequately characterized in clinical trials for single SABA or SAMA agents and for combinations, and patients with COPD must provide a meaningful part of safety data as this population is, in general, more susceptible to systemic safety concerns than are younger asthmatics.

Long-Acting Bronchodilators. As for asthma, these drugs are administered in patients with COPD, as regularly dosed medications to prevent bronchospasm and amelio- 


\section{Drug Development for Asthma AND COPD}

rate symptoms of airflow obstruction, not for relief of acute episodes on an as-needed basis.

LABAs. LABAs are a cornerstone of COPD therapy cleared as maintenance therapies for the relief of air-flow obstruction. This claim is supported by $\mathrm{FEV}_{1}$, typically measured at trough, which is considered a direct efficacy end point that is relevant and meaningful. Additional supportive end points, such as rescue bronchodilator use, COPD exacerbations, or the SGRQ may be included in trials, but the finding of bronchodilatory benefit alone can support this indication. More recently developed LABAs have had to allow for background standard therapy in their placebocontrolled pivotal trials (including LAMA therapy), thus making a trough effect more challenging to demonstrate. More recently cleared LABAs were developed only for COPD, but 2 of the early LABAs (salmeterol as Serevent Diskus and formoterol as Foradil Aerolizer) were developed for both asthma and COPD. The safety concerns that arose for LABAs in asthma have not been shown to apply to COPD, and this may have provided the impetus of subsequent development in this class to focus on COPD alone. Labeling for LABAs, even for those cleared only for COPD, include the boxed warning statement related to asthma.

Phase 2 trials to support dose and regimen are critical to support a LABA clearance for COPD. Many early phase 2 dose-finding trials for LABAs are performed in subjects with asthma, even if the ultimately desired indication is COPD because of the sensitivity of asthmatics to LABA and their ability to discern dose response. Phase 3 trials for the more recently cleared LABAs for COPD have generally continued to evaluate 2 doses of LABA versus placebo and are done in the COPD population.

The NDA for Arcapta Neohaler (indacaterol) provides an important lesson in the use of adaptive trial designs for phase 3 COPD bronchodilator trials, which are sometimes desired in an effort to speed drug development. ${ }^{45}$ The original indacaterol NDA was filed based on a phase 3 adaptive design that determined successful doses at week 2 in an interim analysis. Based on findings at week 2, doses of $150 \mu \mathrm{g} / \mathrm{d}$ and $300 \mu \mathrm{g} / \mathrm{d}$ were carried forward, while lower doses were deemed unsuccessful and were dropped. Upon review, however, the $75-\mu \mathrm{g} / \mathrm{d}$ dose, while dropped in the adaptive design, was still considered a viable dose, as it had just missed the pre-determined cutoff and the drug appeared to show increased efficacy over time. With further study, the $75-\mu \mathrm{g} / \mathrm{d}$ dose was indeed found to be effective and ultimately was the dose cleared for COPD. If adaptive designs are considered, stopping rules for discarding potentially ineffective doses should be conservative, such that only truly unsuccessful doses are dropped. The timing of the data informing the decision for dropping doses should also take into account the time needed to see the full effect of the drug. Because COPD trials for bronchodilators are generally short in duration (12 weeks) and easy to enroll, the risks versus the benefits of an adaptive design approach for dose-finding should be carefully considered.

Indacaterol also provides perspective on how challenging it can be to achieve clearance for 2 dose options of a LABA. The applicant provided justification for the $75-\mu \mathrm{g}$ once-daily dose as well as a $150-\mu \mathrm{g}$ once-daily dose, aiming to demonstrate that the higher dose provided better bronchodilation and symptom control in the more severe subset of COPD subjects. Ultimately, however, only the $75-\mu \mathrm{g}$ dose was cleared as the advantage of the higher dose was not convincingly demonstrated. Achieving clearance of 2 doses of a bronchodilator for COPD is challenging and is currently unprecedented in the United States. However, indacaterol was also cleared at doses of $150 \mu \mathrm{g}$ and $300 \mu \mathrm{g}$ in Europe. The FDA explained their rationale for clearing only the lower dose in an editorial, ${ }^{46}$ noting that the $75-\mu \mathrm{g}$ dose provided for bronchodilation similar to that achieved with the $150-\mu \mathrm{g}$ dose, and that there was no confirmatory trial directly comparing the $75-\mu \mathrm{g}$ and $150-\mu \mathrm{g}$ doses. The FDA will consider 2 doses of a bronchodilator for clearance but a strong case must be made that both doses are necessary, such as the need for a higher dose by a more severe subset of the COPD population.

The safety database for LABAs needs to address the known class risks of hyperglycemia, hypokalemia, and changes in heart rate and heart rhythm. A formal QT safety study is generally needed, and a subset of patients in the phase 3 trials are required to undergo Holter monitoring. The LABAs as a class do prolong the QT interval, but the doses that are cleared have had minimal to no effect on QT. NDA filings for the more recently cleared new-entity LABAs have had extensive safety databases at the time of filing of around 3,000-4,000 COPD subjects exposed to the drug.

LAMAs. LAMAs, like LABAs, are also a cornerstone of COPD therapy, and their development is very similar to that of the LABAs. Careful attention of dose and regimen, with the lowest optimal dose selected for clearance is the rule, and generally applicants exert a great deal of effort in phase 2 trials to determine this, while still studying 2 doses potentially in phase 3 trials. Phase 3 trials for LAMAs, like for LABAs, are generally 12-16 weeks in duration and demonstrate efficacy based on trough $\mathrm{FEV}_{1}$, assessing changes from baseline in each active treatment arm versus placebo. The safety database for LAMAs needs to address the known risks of anticholinergics, specifically dry mouth, urinary retention, changes in heart rate or heart rhythm, and narrow-angle glaucoma risk. The FDA anticipates a robust safety database at the time of FDA filing, similar in scope to that provided for the LABA class. Like LABAs, 
Holter monitor data and a formal QT safety study are required for LAMAs. The LAMA tiotropium provides for some important safety lessons learned.

Spiriva Handihaler (titropium bromide) was the first LAMA cleared for COPD in the United States in January 2004 for the long-term maintenance treatment of bronchospasm associated with COPD, and this drug gained a second indication for reducing COPD exacerbations in 2009. The sponsor, Boehringer Inghelheim, voluntarily notified the FDA in 2007 of a possible new safety concern of stroke. ${ }^{47}$ The pooled data from 29 controlled clinical trials (25 trials with Spiriva HandiHaler, and 4 trials with Spiriva Respimat), reflecting 13,544 subjects with 4,572 personyears exposure, had shown an increased risk ratio for stroke of 1.37 (95\% CI $0.73-2.56$ ) associated with the use of tiotropium. Some of the signal appeared to be driven by the Spiriva Respimat experience. In September 2008, a meta-analysis published in JAMA ${ }^{48}$ analyzed 17 randomized, controlled clinical trials involving ipratropium or tiotropium, assessing the primary combined outcome of cardiovascular death, myocardial infarction, or death, showing a relative risk of 1.58 (95\% CI 1.21-2.06) for inhaled anticholinergics versus placebo. The article concluded that inhaled anticholinergic use for $>30 \mathrm{~d}$ significantly increases the risk of cardiovascular death, myocardial infarction, or stroke in subjects with COPD by approximately 58\%. A second publication in 2008 reinforced this concern. ${ }^{49}$

Ultimately, a 4-y, double-blind, 6,000-subject, placebocontrolled trial for Spiriva HandiHaler (UPLIFT) yielded reassuring results for overall mortality, myocardial infarction, and stroke. ${ }^{50}$ Vital status data were available on $98 \%$ of participants. Mortality occurred in $14.9 \%$ of patients in the tiotropium arm versus $16.5 \%$ in placebo arm, yielding a hazard ratio of 0.89 (95\% CI $0.79-1.02)$. While not sufficiently robust to support a mortality claim favoring the drug, these data were reassuring in light of the former meta-analyses. In UPLIFT, the myocardial infarction rates also favored a tiotropium benefit, being observed in 67 subjects in the tiotropium arm versus 85 subjects in the placebo arm for a relative risk of 0.73 (95\% CI $0.53-1.00$ ), with stroke observed in a balanced fashion in 82 subjects in the tiotropium group versus 80 subjects in the placebo group for a relative risk of 0.95 (95\% CI 0.70-1.29). A 2009 meta-analysis of 19 controlled tiotropium trials included in UPLIFT concluded that tiotropium did not significantly increase the risk of adverse major cardiovascular events among COPD patients. ${ }^{51}$ Additional randomized, controlled data specifically with the tiotropium Respimat device that followed was also reassuring. ${ }^{52}$ The FDA addressed the reassuring safety profile of tiotropium in a publication..$^{53}$ The tiotropium lesson learned is that safety signals from even well-performed meta-analyses may not be valid, and until or unless randomized, controlled data are available, such signals should be interpreted with caution.

Tudorza Pressair (aclidinium bromide) provides for some perspective on the importance of dose/regimen in bronchodilator programs and on the adequacy of a safety database for the COPD indication, with the summary review available on the FDA web site. ${ }^{54}$ The applicant pursued pivotal phase 3 trials with aclidinium $200 \mu \mathrm{g}$ QD, finding an overall statistically significant difference of approximately $60 \mathrm{~mL}$ in trough $\mathrm{FEV}_{1}$. Upon reviewing the data, the FDA did not clear the drug, instead recommending study of higher doses and more frequent dosing regimens given the relatively limited effects seen on $\mathrm{FEV}_{1}$. A resubmission to support aclidinium at $400 \mu \mathrm{g}$ twice daily was successful. This experience underscores the importance of adequate dose finding prior to advancing to phase 3 studies. The adequacy of a safety database was also an issue for this application. While many studies had been performed at various dose levels, the subject exposure at the cleared dose of $400 \mu \mathrm{g}$ twice daily dose only marginally exceeded the International Council for Harmonization guideline minimums for a chronic use drug, with 1,471 subjects exposed, of whom 733 subjects were exposed for 6 months or longer and 329 subjects for $1 \mathrm{y}$ or longer. The FDA observed the database provided for infrequent serious outcomes, sometimes with imbalances (albeit small numbers overall) and concluded that the database, while acceptable for clearance nonetheless required a phase 4 study to further understand the safety outcomes of aclidinium, particularly its cardiovascular safety. The ASCENT study, evaluating the effect of aclidinium bromide on long-term cardiovascular safety and exacerbations in patients with moderate to very severe COPD, is currently fully enrolled and ongoing. ${ }^{55}$ Thus, while International Council for Harmonization guidelines are a framework for an adequate safety database, most applicants exceed these guidelines in developing drugs for asthma and COPD.

Combination LAMA/LABA Therapy. The same general tenets for SAMA/SABA combination drugs apply to a combination LAMA/LABA program. A full understanding of each single agent's benefit as a bronchodilator is expected even if the single agents are not being considered for market. It is not required that each mono-component be cleared for COPD, but if they are not, they should be fully characterized as to dose and regimen. All studies should be performed with the same drug delivery device, and there must be no evidence of pharmaceutical interaction (in vitro or in vivo) for the combination versus the single ingredients within the device. PK data are needed to substantiate the lack of a pharmaceutical interaction for the combination versus the single ingredients. Pharmacodynamic data can also be provided to assure that, in general, the bron- 
chodilatory benefit provided by the combined product represents approximately the sum of the individual LAMA and LABA mono-components. This has generally been the case with LAMA/LABA combinations, as each drug affects different receptors in the airway, providing bronchodilation in a different way. When a full understanding of the single drug components is achieved and the lack of pharmaceutical interactions is established, the traditional phase 3 factorial design trials can be completed to establish efficacy for the combination versus placebo and versus each single component. Placebo is generally included in such studies to adequately inform the safety profile of the LAMA/LABA.

The primary efficacy end point for demonstrating the benefit of the LAMA is often the full $\mathrm{FEV}_{1}$ area under the curve throughout the entire dosing interval, while the LABA's benefit is often measured as the trough $\mathrm{FEV}_{1}$ change from baseline. For Utibron Neohaler (indacaterol [IND]/glycopyrollate [GP]), however, the same primary efficacy end point of 12-hour $\mathrm{FEV}_{1}$ area under the curve was used to support the benefit of the combination versus the mono-components, as per the summary FDA review. ${ }^{56}$ Utibron Neohaler (cleared as glycopyrollate $15.6 \mu \mathrm{g}$ twice daily and indacaterol $27.5 \mu \mathrm{g}$ twice daily) provides some helpful perspective on the development path for a LABA/LAMA. Prior to Utibron's clearance both single ingredients had been cleared by the FDA; the Seebri Neohaer (glycopyrollate $15.6 \mu \mathrm{g}$ twice daily) was cleared by the FDA during their review of the Utibron Neohaler application, and the Arcapta Neohaler (indacaterol new drug $75 \mu \mathrm{g}$ once daily) had been cleared since 2011. Each drug was well understood for COPD, but one was cleared for twice-daily use while the other had been cleared for once-daily use. A unique challenge thus posed by combining these 2 products was to discern which regimen (once daily or twice daily) best fit the combination. Although the applicant began with a dose-finding study of once-daily glycopyrollate in an attempt to match the oncedaily indacaterol, it was eventually determined that it was more appropriate to select a twice-daily dose of indacaterol to match the twice-daily glycopyrollate.

The Utibron Neohaler application supported mention of SGRQ results in labeling. In both pivotal studies, the proportions of subjects with a change from baseline of 4 (the minimal clinical important difference) were greater for the Utibron Neohaler than for placebo. The Utibron Neohaler application also provided some perspective on QT safety. A formal QT safety study was marginally positive for Utibron Neohaler, although the dose evaluated was 16fold higher for investigational new drug and 32-fold higher for GP; even at this dose, the mean maximal change in QT at 30 min post-dose (presumably peak) was $8.70 \mathrm{~ms}(90 \% \mathrm{CI}$ 7.3-10.1). Per the Utibron label, "although a marginal QT effect of Utibron Neohaler was observed at the suprath- erapeutic dose, it is unlikely there will be a clinically relevant effect at the therapeutic exposure." 57

Combination ICS/LABA Therapy. Three ICS/LABA therapies are cleared for COPD: Advair Diskus 250/50, containing fluticasone propionate and salmeterol xinofoate; Symbicort, containing budesonide and formoterol fumarate; and Breo Ellipta, containing fluticasone furoate (FF) and vilanterol trifenatate (VI). All are cleared at a single fixed dose of ICS and LABA. ICS mono-therapy is not FDA-cleared for COPD. ICS/LABA programs need to substantiate the benefit of the LABA component for COPD, which is provided by assessing $\mathrm{FEV}_{1}$ benefit. In all cases so far, the LABA dose for the ICS/LABA has been identical to that for the cleared standalone LABA therapy. The more challenging aspect of an ICS/LABA program for COPD is the need to substantiate the benefit of the inhaled steroid component, and to clarify that with adequate dose exploration. ICS have generally shown modest benefits on $\mathrm{FEV}_{1}$ in the COPD population, with little evidence for dose response. $\mathrm{FEV}_{1}$ is an acceptable primary efficacy end point for the benefit offered by the ICS. However, interest in the more clinically relevant effect of ICS on reducing COPD exacerbations has led to this being a primary efficacy end point and an important additional claim for the combination ICS/LABA products. A pneumonia risk is noted with respect to all inhaled steroids in all ICS/LABA labels when used for COPD as part of class labeling. This same risk has not been observed for asthma.

Breo Ellipta (FF $100 \mu \mathrm{g} / \mathrm{VI} 25 \mu \mathrm{g}$ ), is the most recently cleared ICS/LABA therapy for COPD and provides some helpful lessons on obtaining a COPD-exacerbation claim. There were 2 replicate pivotal trials performed, both lasting 52 weeks. Each study included $3 \mathrm{FF} / \mathrm{VI}$ dosage arms (50/25, 100/25, and 200/25), as well as a VI25 dosage arm. The goal of each pivotal trial was to assess the proper strength of FF that would provide a reduction in COPD exacerbations. The primary efficacy end point was the annualized rate of moderate and severe exacerbations. A hierarchical analysis of the 3 FF/VI arms (200/25, 100/25, and 50/25) was planned, and step-down to the lower dose could only occur if the higher dose was successful. In one pivotal trial, the highest strength did not meet significance, such that the medium strength of 100/25 could only be considered nominally significant. With that said, the 100/25 strength provided for a $34 \%$ reduction in moderate to severe exacerbations over vilanterol. The second trial made significance at the highest strength as well as at the medium strength, which showed a $21 \%$ reduction in moderate to severe COPD exacerbations that was statistically significant over vilanterol. The FDA considered the totality of data and cleared a COPD-exacerbation claim for the FF/VI 100/25 strength, despite one trial failing due to a prespecified hierarchical analysis. As per the medical of- 
ficer review: "While the two 52-week exacerbation trials do not provide replicate evidence of statistically significant improvement based on the testing hierarchy for FF/VI 100/25 over VI25, the totality of the efficacy data comparing FF/VI 100/25 to VI25 demonstrates the benefit added by the steroid component to the combination product." 58

Combination ICS/LAMA/LABA Therapy. Trelegy Ellipta is a combination of fluticasone furoate (an ICS), umeclidinium (a LAMA), and vilanterol (a LABA) that was cleared in 2017 for the long-term, once-daily, maintenance treatment of COPD patients. Trelegy is cleared at a fixed dose of fluticasone furoate/umeclidinium/vilanterol of $100 / 62.5 / 25 \mu \mathrm{g}$ once daily. The indication is uniquely worded to apply to COPD patients who are already on fluticasone furoate/vilanterol (Breo Ellipta, GlaxoSmithKline, Middlesex, United Kingdom) for reducing COPD exacerbations and treating air-flow obstruction, in whom additional bronchodilatory benefit is desired (offered by the LAMA, umeclidinium). The restricted indication is due to the fact that the primary efficacy data provided for Trelegy focused on the added bronchodilatory benefit offered by the LAMA over the ICS/LABA. Trelegy is also indicated for COPD patients already receiving the triple therapy via 2 different inhalers (Breo Ellipta and Incruse Ellipta). The indication is therefore specific to patients already taking drugs that are already part of Trelegy. Labeling does not currently address COPD exacerbations, but in September 2017 partners GlaxoSmithKline and Innoviva released the results of the phase 3 IMPACT study (InforMing the Pathway of COPD Treatment), in which Trelegy Ellipta reduced COPD exacerbation rates significantly when compared to Breo Ellipta (a 15\% reduction was observed) and Anoro Ellipta (a 25\% reduction was observed). ${ }^{59}$ It is possible that the results of IMPACT may support a broader indication for Trelegy Ellipta. The development of Trelegy for COPD followed the full development and clearances of the single LABA and LAMA agents, the dual LAMA/LABA agent, and the dual ICS/LABA agent, all delivered in the same device. Trelegy Ellipta's demonstration of a benefit on COPD exacerbations versus Breo Ellipta and Anoro Ellipta may allow for a broader indication. Each agent for COPD (alone or in dual combinations) was well understood prior to combining all 3 agents in a triple-therapy approach, with all drugs delivered via the same device. Multiple large studies were required to support the first triple-combination inhaled drug therapy for a limited COPD population.

Two pivotal 12-week trials demonstrated the added bronchodilatory benefit of the LAMA (umeclidinium) when added to the combination of the ICS/LABA (fluticasone furoate/vilanterol). Each pivotal trial demonstrated an approximate gain in trough $\mathrm{FEV}_{1}$ of $120 \mathrm{~mL}$ attributable to the LAMA. More subjects treated with the triple therapy in each study also responded to the SGRQ with at least a 4-point change from baseline. The pivotal trials did not support the benefit of the ICS (fluticasone furoate) versus the LAMA/LABA (umeclidinium/vilanterol), but instead referred to the earlier Breo Ellipta studies in which a benefit of fluticasone furoate was established over vilanterol alone for COPD exacerbations. Importantly, the Trelegy Ellipta label (like all products containing LABA) contains the usual boxed warning for asthma-related deaths, even though the indicated use is for COPD; the additional text conveys that Trelegy has not been studied in asthma and is not indicated for asthma.

Phosphodiesterase Inhibitors and Xanthines. The phosphodiesterase 4 (PDE-4) inhibitor cilomilast did not gain FDA clearance yet it was the first promising PDE-4 inhibitor to be evaluated for COPD, with results summarized in a 2008 publication. ${ }^{60}$ In the 5 phase 3 trials performed, the mean change from baseline in $\mathrm{FEV}_{1}$ in the cilomilast group versus placebo ranged from a modest $24 \mathrm{~mL}$ to $44 \mathrm{~mL}$. Additionally, the effect of cilomilast versus placebo on SGRQ was generally similar between arms, with only 1 study showing a significant benefit that met the minimal clinically important difference. In the lone study specifically designed to evaluate COPD exacerbations, no benefit was seen. In all studies, GI adverse events were also reported more frequently, a recognized class effect. Due to these disappointing results, the development of cilomilast for COPD was terminated.

Daliresp (roflumilast) is a PDE-4 inhibitor that initially sought clearance by exploring bronchodilatory benefits in COPD, which were modest on the order of $50 \mathrm{~mL}$ versus placebo. As trials progressed, an effect of Daliresp on COPD exacerbations was appreciated as more severe COPD subjects were studied. The clearance of Daliresp was based on its ability to reduce COPD exacerbations in the severe subset of COPD subjects who had a history of exacerbations. An additional clarification learned during the development of Daliresp was that the patients who contributed the most to the COPD exacerbation signal were those with chronic bronchitis. The Daliresp development program involved many studies in subjects with COPD prior to defining the most responsive phenotype. The primary safety concerns with the PDE inhibitors is GI tolerability, but preclinical concerns with vasculitis and spermatogenesis may arise for this class as well.

Theophylline preparations are still used for the management of COPD, but their clearances are dated, with many drug formulations being discontinued. Due to toxicity concerns as well, theophylline preparations have not generally been pursued for COPD. 


\section{Generic Inhaled Drug Development for COPD and Asthma}

The FDA Website lists a total of seventeen productspecific draft guidances (2013-2016 release dates) related to developing an acceptable generic inhaled drug product. ${ }^{61}$ The drugs listed include older agents, such as albuterol sulfate, levalbuterol tartrate, budesonide, beclomethasone dipropionate, and ipratropium bromide, as well as combination ICS/LABA agents such as fluticasone propionate/salmeterol xinofoate and budesonide/formoterol fumarate. However, more recently cleared agents also have FDA draft guidances regarding their generic development: ciclesonide, fluticasone furoate, fluticasone furoate/vilanterol trefenatate, formoterol fumarate/mometasone furuoate, mometasone furoate, and umeclidinium bromide. Thus, ICS, LABA, and LAMA drugs, as well as combination ICS/LABA drugs, have FDA draft guidances addressing generic development.

Despite the many guidances, generic clearances for inhaled drug DPIs or pMDIs are, with the exception of albuterol or levalbuterol, still pending as of July 2017. The guidances rely heavily on chemistry/manufacturing and in vitro performance data to support clearance because clinical studies are often limited in detecting differences in drug delivery to the site of action. Many quantitative in vitro tests must be performed to demonstrate that the proposed generic inhaled drug is being delivered in an equivalent manner compared to the reference product. The singleactuation emitted dose as well as the particle-size distribution of the product must be tested at various stages of the life cycle of the product and at varying flows, and the dose must consistently reach a bioequivalence standard for sameness. The drug delivery system should be very similar if not identical to the reference device, involving the same number and type of steps for successful use. If the reference product, for instance, includes a passive breath-actuated device, with 60 pre-metered doses available and 4 steps for inhalation, then the new generic device should meet these same criteria, be of similar size and shape, and provide for comparable device resistance for successful actuation. Sponsors are encouraged to submit a working model and engineering drawings of the product to the Office of Generic Drugs prior to submitting their application to assure acceptability. A bioequivalent PK crossover study is necessary to show equivalence based on area under the curve and peak serum concentration, with geometric mean test/reference ratios falling within $80-125 \%$ for $90 \%$ CI. These studies, while reasonably likely to be successful, can be demanding if the reference product itself provides for variable PK profiles. The bioequivalence PK studies support equivalent systemic exposure of the products (and therefore systemic safety), but they cannot be used to ex- trapolate efficacy as bioequivalence for inhaled drugs is determined by delivery to the site of action, which is the airways, not the circulation. Therefore, a clinical end point study that compares the test versus reference products and establishes equivalence based on the test/reference ratio for the primary efficacy end point(s) is also required, and the results must fall within $80-125 \%$ for $90 \%$ CI (except for albuterol). For a SABA, LABA, LAMA, and ICS, this primary end point has been $\mathrm{FEV}_{1}$, although it is assessed in different ways for each product. These clinical studies can be quite challenging to conduct due to the requirement to demonstrate that the confidence intervals indeed lie within $80-125 \%$ on a variable end point. Hence, the development of generic inhaled drugs has proven challenging, but many programs are underway.

\section{Summary}

The regulatory clearance process is often criticized for being too slow or, if safety concerns arise, too permissive. Confusion exists when the FDA has not yet cleared a therapy, but "everyone is recommending it and using it." The development of drugs under the FDA's purview is informed by years of regulatory experience and precedence, as well as very detailed reviews of data. The evidence needed to inform clinical practice guidelines is not the same as the type of evidence needed for FDA clearance. Through the examples provided, it is hoped that there is a better understanding of the regulatory clearance process for drugs to treat asthma and COPD.

\section{REFERENCES}

1. Pharmaceutical Research and Manufacturers of America. Biopharmaceutical research and development: the process behind new medicines. 2015. http://phrma-docs.phrma.org/sites/default/files/pdf/rd brochure_022307.pdf Accessed December 27, 2017.

2. United States Food and Drug Administration. Guidances (drugs). https://www.fda.gov/Drugs/GuidanceComplianceRegulatoryInforma tion/Guidances/default.htm Accessed December 27, 2017.

3. United States Food and Drug Administration. Guidance for industry. Providing clinical evidence of effectiveness for human drug and biological products. May 1998. https://www.fda.gov/downloads/ drugs/guidancecomplianceregulatoryinformation/guidances/ ucm072008.pdf Accessed December 27, 2017.

4. United States Food and Drug Administration. Non-inferiority clinical trials to establish effectiveness: guidance for industry. November 2016. https://www.fda.gov/downloads/Drugs/Guidances/ UCM202140.pdf Accessed December 27, 2017.

5. United States Food and Drug Administration. Guidance for industry. Expedited programs for serious conditions - drugs and biologics. May 2014. https://www.fda.gov/downloads/drugs/guidancecomplia nceregulatoryinformation/guidances/ucm358301.pdf Accessed December 27, 2017.

6. United States Food and Drug Administration. Novel drugs summary 2016. https://www.fda.gov/drugs/developmentapprovalprocess/ druginnovation/ucm534863.htm Accessed December 27, 2017. 


\section{Drug Development for Asthma AND COPD}

7. Katial RK, Bensch GW, Busse WW, Chipps BE, Denson JL, Gerber AN, et al. Changing paradigms in the treatment of severe asthma: the role of biologic therapies. J Allergy Clin Immunol 2017;5(2 Suppl): S1-S14.

8. Nordenberg T; United States Food and Drug Administration. CFCfree medication for an ailing ozone layer. December 2015. https:// www.fda.gov/drugs/resourcesforyou/ucm085278.htm Accessed December 27, 2017.

9. United States Food and Drug Administration. Application no. 21730 medical reviews. March 2005. https://www.accessdata.fda.gov/ drugsatfda_docs/nda/2005/021730s000_MedR.pdf Accessed December 27, 2017.

10. Nelson H, Weiss ST, Bleeker ER, Yancey SW, Dorinsky PM; SMART study group. The Salmeterol Multicenter Asthma Research Trial: a comparison of usual pharmacotherapy for asthma or usual pharmacotherapy plus salmeterol. Chest 2006;129(1):15-26.

11. Mann M, Chowdhury B, Sullivan E, Nicklas R, Anthracite R, Meyer RJ. Serious asthma exacerbations in asthmatics treated with highdose formoterol. Chest 2003;124(1):70-74.

12. Chowdhury BA, Seymour SM, Levenson MS. Assessing the safety of adding LABAs to inhaled corticosteroids for treating asthma. N Engl J Med 2011;364(26):2473-2475.

13. Stempel DA, Raphiou IH, Kral KM, Yeakey AM, Emmett AH, Prazma CM, et al. Serious asthma events with fluticasone plus salmeterol versus fluticasone alone. N Engl J Med 2016;374(19):18221830.

14. Stempel DA, Szefler SJ, Pedersen S, Zeiger RS, Yeakey AM, Lee LA, et al. Safety of adding salmeterol to fluticasone propionate in children with asthma. N Engl J Med 2016;375(9):840-849.

15. Peters SP, Bleeker ER, Canonica GW, Park BY, Ramirez R, Hollis $\mathrm{S}$, et al. Serious asthma events with budesonide plus formoterol vs budesonide alone. N Engl J Med 2016;375(9):850-860.

16. Merck. Press release announcing top line results from long-term LABA safety study of Dulera inhalation aerosol. May 22, 2017. http://www.mrknewsroom.com/news/company-statements/merckannounces-top-line-results-long-term-laba-safety-study-duleramometaso Accessed December 27, 2017.

17. Boehringer Ingelheim Pharmaceuticals. Spiriva Respimat full prescribing information. https://www.accessdata.fda.gov/drugsatfda docs/label/2015/207070s000lbl.pdf Accessed December 27, 2017.

18. Kerstjens HAM, Engel M, Dahl R, Paggiaro P, Beck E, Vandewalker $\mathrm{M}$, et al. Tiotropium in asthma poorly controlled with standard combination. N Engl J Med 2012;367(13):1198-1207.

19. United States Food and Drug Administration. Approval NDA 207070/ Original 1. https://www.accessdata.fda.gov/drugsatfda_docs/appletter/ 2015/207070Orig1s0001tr.pdf Accessed December 27, 2017.

20. Bjermer L, Alving K, Diamant Z, Magnussen H, Pavord I, Piacentini $\mathrm{G}$, et al. Current evidence and future research needs for FeNO measurement in respiratory diseases. Respir Med 2014;108(6):830-841.

21. Temple RJ, Meyer RJ. Continued need for placebo in many cases, even when there is effective therapy. Arch Intern Med 2003;163(3): 371-373.

22. United States Food and Drug Administration. Guidance for industry. Orally inhaled and intranasal corticosteroids: evaluation of the effects on growth in children. March 2007. https://www.fda.gov/down loads/Drugs/GuidanceComplianceRegulatoryInformation/ Guidances/UCM071968.pdf Accessed December 27, 2017.

23. Marcello C, Carlo L. Asthma phenotypes: the intriguing selective Intervention with montelukast. Asthma Res Pract 2016;2:11.

24. Merck. Singulair full prescribing information. June 2016. http://www. merck.com/product/usa/pi_circulars/s/singulair/singulair_pi.pdf $A c$ cessed December 27, 2017.
25. Murphy KR, Bender BG. Treatment of moderate to severe asthma: patient perspectives on combination inhaler therapy and implications for adherence. J Asthma Allergy 2009;2:63-72.

26. Strunk RC, Bloomberg GR. Omalizumab for asthma. N Engl J Med 2006;354(25):2289-2295.

27. Mitchell PD, El-Gammal AI, O’Byrne PM. Emerging monoclonal antibodies as targeted innovative therapeutic approaches to asthma. Clin Pharmacol Ther 2016;99(1):38-48.

28. Menzella F, Lusuardi M, Montanari G, Galeone C, Facciolongo N, Zucchi L. Clinical usefulness of mepolizumab in severe eosinophilic asthma. Ther Clin Risk Manag 2016;12:907-916.

29. Nair P, Pizzichini MM, Kjarsgaard M, Inman MD, Efthimiadis A, Pizzichini E, et al. Mepolizumab for prednisone dependent asthma with sputum eosonophilia. N Engl J Med 2009;360(10):985-993.

30. Ortega HG, Liu MC, Pavord ID, Bruselle GG, FitzGerald JM, Chetta A, et al. Mepolizumab in patients with severe eosinophilic asthma. N Engl J Med 2014;371(13):1198-1107.

31. Castro M, Zangrilli J, Weschler ME, Bateman ED, Grusselle GG, Bardin P, et al. Reslizumab for inadequately controlled asthma with elevated blood eosinophil counts: results from two muticentre, parallel, double-blind, randomised, placebo-controlled, phase 3 studies. Lancet Respir Med 2015;3(5):355-366.

32. Bagnasco D, Ferrando M, Varricchi G, Passalacqua G, Canonica GW. A critical evaluation of anti IL-13 and anti IL-4 strategies in severe asthma. Int Arch Allergy Immunol 2016;170(2):122-131.

33. United States Food and Drug Administration. Chronic obstructive pulmonary disease: developing drugs for treatment. Draft guidance for industry. May 2016. http://www.fda.gov/downloads/drugs/guid ancecomplianceregulatoryinformation/guidances/ucm071575.pdf Accessed December 27, 2017.

34. GlaxoSmithKline. Advair Diskus full prescribing information. February 2017. https://www.accessdata.fda.gov/drugsatfda_docs/label/ 2017/021077s0581bl.pdf Accessed December 27, 2017.

35. Casaburi R, Celli B, Crapo J, Criner G, Croxton T, Gaw A, et al. The COPD Biomarker Qualification Consortium (CBQC). J COPD 2013; 10(3):367-377.

36. Mannino DM, Tal-Singer R, Lomas DA, Vestbo J, Barr G, Tetzlaff $\mathrm{K}$, et al. Plasma fibrinogen as a biomarker for mortality and hospitalized exacerbations in people with COPD. J COPD 2015; 2(1):23-34

37. Vestbo J, Anderson W, Coxson HO, Crim C, Dawber L, Edwards L, et al. Evaluation of COPD Longitudinally to Identify Predictive Surrogate Endpoints (ECLIPSE). Eur Respir J 2008;31(4):869-873.

38. United States Food and Drug Administration. Draft. Qualification of biomarker - plasma fibrinogen in studies examining exacerbations and/or all-cause mortality in patients with chronic obstructive pulmonary disease. http://www.fdanews.com/ext/resources/files/07-15/07-07-15-plasmafibrinogen.pdf?1486936442 Accessed December 27, 2017.

39. Donohue JF. Minimal clinically important differences in COPD lung function. COPD 2005;2(1):111-124.

40. Jones PW. St George's Respiratory Questionnaire: MCID. COPD 2005;2(1):75-79.

41. Meguro M, Barley EA, Spencer S, Jones PW. Development and validation of an improved COPD-specific version of the St. George Respiratory Questionnaire. Chest 2007;132(2):456-463.

42. Wijkstra PJ, TenVergert EM, Van Altena R, Otten V, Postma DS, Kraan J, Koeter GH. Reliability and validity of the Chronic Respiratory Questionnaire. Thorax 1994;49(5):465-467.

43. United States Food and Drug Administration. Clinical outcome assessment compendium. Version 1. December 2015. http://www.fda.gov/ downloads/Drugs/DevelopmentApprovalProcess/DevelopmentReso urces/UCM481225.pdf Accessed December 27, 2017.

44. Evidra. EXACT-PRO initiative. March 2016. http://www.exactpro initiative.com Accessed December 27, 2017. 


\section{Drug Development for Asthma AND COPD}

45. United States Food and Drug Administration. Application no. 022383Orig 1s000 summary review. July 2011. https://www.access data.fda.gov/drugsatfda_docs/nda/2011/022383Orig1s000SumR.pdf Accessed December 27, 2017.

46. Chowdhury BA, Seymour SM, Michele TM, Durmowicz AG, Liu D, Rosebraugh CJ. The risks and benefits of indacaterol: the FDA's review. N Engl J Med 2011;365(24):2247-2249.

47. Food and Drug Administration. Approval packages for application no. 21-395/S029 Spiriva HandiHaler. December 2009. https://www. accessdata.fda.gov/drugsatfda_docs/nda/2009/021395Orig1s029.pdf Accessed December 27, 2017.

48. Singh S, Like YK, Furberg CD. Inhaled anticholinergics and risk of major adverse cardiovascular events in patients with chronic obstructive pulmonary disease: a systematic review and meta-analysis. JAMA 2008;300(12):1439-1450.

49. Lee TA, Pickard AS, Au DH, Bartle B, Weiss KB. Risk for death associated with medications for recently diagnosed chronic obstructive pulmonary disease. Ann Intern Med 2008;149(6):380-390.

50. Tashkin DP, Celli B, Senn S, Burkhart D, Kesten S, Menjoge S, et al. A 4-year trial of tiotropium in chronic obstructive pulmonary disease. N Engl J Med 2008;359(15):1543-1554.

51. Rodrigo GJ, Castro-Rodriguez JA, Nannini LJ, Plaza Moral V, Schiavi EA. Titropium and risk for fatal and nonfatal cardiovascular events in patients with chronic obstructive pulmonary disease: systematic review with meta-analysis. Respir Med 2009;103(10):1421-1429.

52. Wise RA, Anzueto A, Cotton D, Dahl R, Devins T, Disse B, et al. Tiotropium Respimat inhaler and the risk of death in COPD. N Engl J Med 2013;369(16):1491-1501.

53. Michele TM, Pinheiro S, Iyasu S. The safety of tiotropium: the FDA's conclusions. N Engl J Med 2010;363(12):1097-1099.

54. United States Food and Drug Administration. Application no. 202450Orig1s000 summary review. July 2012. https://www.accessdata.
fda.gov/drugsatfda_docs/nda/2012/202450Orig1s000SumR.pdf Accessed December 27, 2017.

55. Evaluate the effect of aclidinium bromide on long-term cardiovascular safety and exacerbations in moderate to very severe COPD patients (ASCENT COPD). https://clinicaltrials.gov/ct2/show/ NCT01966107 Accessed December 27, 2017.

56. United States Food and Drug Administration. Application no. 207930Orig1s000 summary review. October 2015. https://www. accessdata.fda.gov/drugsatfda_docs/nda/2015/207930Orig1s000SumR. pdf Accessed December 27, 2017.

57. Novartis Pharmaceuticals. Utibron Neohaler full prescribing information. January 2017. https://www.accessdata.fda.gov/drugsatfda docs/label/2017/207930s0021bl.pdf Accessed December 27, 2017.

58. United States Food and Drug Administration. Application no. 204275Orig1s000 medical reviews. https://www.accessdata.fda.gov/ drugsatfda_docs/nda/2013/204275Orig1s000MedR.pdf Accessed December 27, 2017.

59. GlaxoSmithKline. GSK and Innoviva report positive headline results from IMPACT study showing single inhaler triple therapy Trelegy Ellipta reduced COPD exacerbations. September 2017. https:// us.gsk.com/en-us/media/press-releases/2017/gsk-and-innovivareport-positive-headline-results-from-impact-study-showing-singleinhaler-triple-therapy-trelegy-ellipta-reduced-copd-exacerbations/ Accessed December 27, 2017.

60. Rennard S, Knobil K, Rabe KF, Morris A, Schachter N, Locantore $\mathrm{N}$, et al. The efficacy and safety of cilomilast in COPD. Drugs 2008;68(2 Suppl):3-57

61. United States Food and Drug Administration. Product-specific guidances for generic drug development. October 2017. https://www.fda. gov/Drugs/GuidanceComplianceRegulatoryInformation/Guidances/ ucm075207.htm Accessed December 27, 2017.

\section{Discussion}

Pleasants: When you talk about 80 $125 \%$ bioequivalence with generics, is it blood levels or drug delivery? How do you define that $80-125 \%$ bioequivalence?

Mann: When we talk about 80$125 \%$ bioequivalence for generic drugs there are 3 different categorical endpoints in which you have to be in that $80-125 \%$ range. The first is the chemistry manufacturing and controls (CMC), which are sort of the performance data of the drug. How it works, emitted dose, and particle size distribution, MMAD, yes. The guidances really want these $\mathrm{CMC}$ characteristics to be very dead-on to the reference product. There's a reason why they're doing that. There is generally, even precedence at the FDA, that if you change your manufacturing site, you have to support that you are making the same drug, and the CMC challenges there are significant. With that in mind, the FDA wants a generic inhaled drug to also be very much the same from a CMC standpoint. The 80$125 \%$ is the standard that must be met for emitted dose, and for the emitted dose broken down by particle size distributions. To be clear, also, it is not that the drug needs to be within 80$125 \%$ for the point estimates, these are confidence intervals. The point estimates generally need to be very close to $1: 1$ ratios for the innovator versus the reference. It's very difficult. Your generic inhaled drug really does need to look pretty much exactly like the reference product for all of these characteristics. CMC is very challenging. The next big category is pharmacokinetics, and here we are looking at area under the curve and the maximal concentration $\left(\mathrm{C}_{\max }\right)$. Your ratios again for each of these comparisons have to be almost 1:1 so that you fall within $80-125 \%$ confidence. The third category the FDA adds is some kind of pharmacodynamic endpoint. Because these inhaled drugs work topically, the FDA needs a pharmacodynamic endpoint as well. For LABAs or albuterol it's usually trough $\mathrm{FEV}_{1}$. And again, you need to demonstrate very similar $\mathrm{FEV}_{1}$ changes for your new generic drug versus the reference drug-the ratio has to be within $80-125 \%$ for the confidence intervals. It should be for instance a $1: 1$ ratio with confidence intervals that are no less than 0.8 and no more than 1.25 . For inhaled steroids it's also trough $\mathrm{FEV}_{1}$. So the FDA has these 3 different categories where you need to establish bioequivalence and you have to nail it, nail it, nail it. If you want to ask me why don't we have a generic yet? I think 
when you do so many tests and always have to be within $80-125 \%$, it's challenging.

MacIntyre: This may be obvious to everybody but me. Why do you call them non-inferiority studies and not comparable studies?

Mann: The word comparable doesn't have a strong regulatory definition around it. It just means generally similar and it doesn't have a statistical or regulatory connotation. But non-inferiority has the statistical connotation of an accepted boundary of similarity that is shown with a fairly tight degree of confidence. I think the term non-inferiority has the statistical connotation, which gives it much more meaning and relevance, while comparability. . . well, it's just comparable. The results look similar, but without the mathematical significance.

Peters: I'm curious, if a drug has been on the market in Europe for many years and has shown benefit and safety in a large number of patients, does the FDA ever change the regulations or the order of requirements to do toxicology again in animals?

Mann: Yes. If a sponsor came in with something that has a wealth of human experience where safety is understood, either from clinical trials done in Europe or from the marketing experience, I do think the FDA will work with you and possibly reduce the need for certain animal studies. They will work with you on a very reasonable pre-clinical plan to address the most important concerns. A key concern for a lot of products is carcinogenicity. Those are the most expensive studies for sponsors to do - and for a chronically administered drug, it is generally desired to determine if there is any carcinogenic potential. Unfortunately, as you can imagine, even if a drug has been used by millions of people and we haven't seen cancer it's not very reassuring. The agency might still mandate certain studies, but they'll work with you to try to reduce your regulatory burden as much as possible.

MacIntyre: Thank you, this was fascinating. 